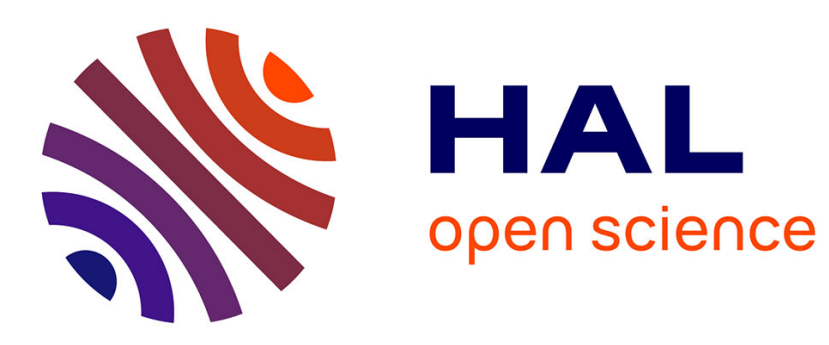

\title{
On the Robustness of the Snell envelope
}

Pierre del Moral, Peng Hu, Nadia Oudjane, Bruno Rémillard

\section{To cite this version:}

Pierre del Moral, Peng Hu, Nadia Oudjane, Bruno Rémillard. On the Robustness of the Snell envelope.

[Research Report] RR-7303, INRIA. 2010, pp.41. inria-00487103v4

\section{HAL Id: inria-00487103 https://hal.inria.fr/inria-00487103v4}

Submitted on 15 Jan 2011

HAL is a multi-disciplinary open access archive for the deposit and dissemination of scientific research documents, whether they are published or not. The documents may come from teaching and research institutions in France or abroad, or from public or private research centers.
L'archive ouverte pluridisciplinaire HAL, est destinée au dépôt et à la diffusion de documents scientifiques de niveau recherche, publiés ou non, émanant des établissements d'enseignement et de recherche français ou étrangers, des laboratoires publics ou privés. 


\section{N R I A}

INSTITUT NATIONAL DE RECHERCHE EN INFORMATIQUE ET EN AUTOMATIQUE

\section{On the Robustness of the Snell envelope}

Pierre Del Moral, Peng Hu, Nadia Oudjane, Bruno Rémillard

\section{$\mathbf{N}^{\circ} \mathbf{7 3 0 3}$}

May 2010

Stochastic Methods and Models

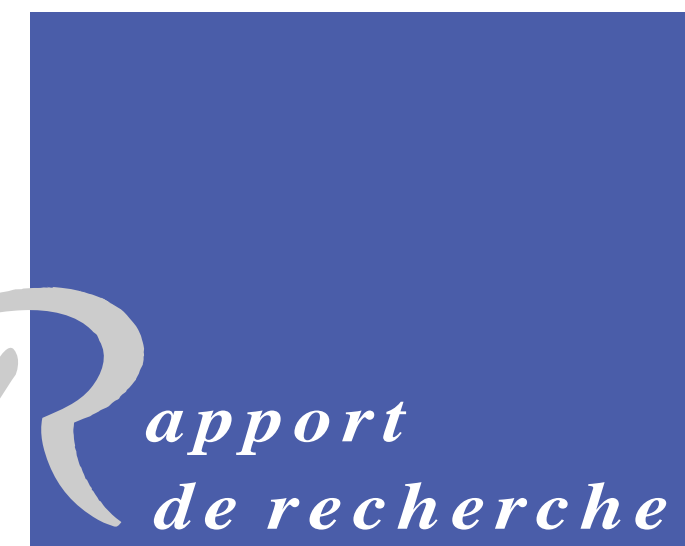





\title{
On the Robustness of the Snell envelope
}

\author{
Pierre Del Moral*, Peng Hu丹, Nadia Oudjant团, Bruno Rémillard § \\ Theme : Stochastic Methods and Models \\ Applied Mathematics, Computation and Simulation \\ Équipe-Projet ALEA
}

Rapport de recherche $\mathrm{n}^{\circ} 7303$ - May 2010 - 41 pages

\begin{abstract}
We analyze the robustness properties of the Snell envelope backward evolution equation for the discrete time optimal stopping problem. We consider a series of approximation schemes, including cut-off type approximations, Euler discretization schemes, interpolation models, quantization tree models, and the Stochastic Mesh method of Broadie-Glasserman. In each situation, we provide non asymptotic convergence estimates, including $\mathbb{L}_{p}$-mean error bounds and exponential concentration inequalities. We deduce these estimates from a single and general robustness property of Snell envelope semigroups. In particular, this analysis allows us to recover existing convergence results for the quantization tree method and to improve significantly the rates of convergence obtained for the Stochastic Mesh estimator of Broadie-Glasserman.

In the second part of the article, we propose a new approach using a genealogical tree approximation of the reference Markov process in terms of a neutral type genetic model. In contrast to Broadie-Glasserman Monte Carlo models, the computational cost of this new stochastic particle approximation is linear in the number of sampled points. Some simulations results are provided and confirm the interest of this new algorithm.
\end{abstract}

Key-words: Snell envelope, optimal stopping, American option pricing, genealogical trees, interacting particle model

\footnotetext{
* Centre INRIA Bordeaux et Sud-Ouest \& Institut de Mathématiques de Bordeaux , Université de Bordeaux I, 351 cours de la Libération 33405 Talence cedex, France, Pierre.DelMoral@inria.fr

$\dagger$ Centre INRIA Bordeaux et Sud-Ouest \& Institut de Mathématiques de Bordeaux , Université de Bordeaux I, 351 cours de la Libération 33405 Talence cedex, France, Peng.Hu@inria.fr

‡ EDF R \& D Clamart (nadia.oudjane@edf.fr)

$\S$ HEC Montréal (bruno.remillard@hec.ca)
}

Centre de recherche INRIA Bordeaux - Sud Ouest 


\section{Sur la robustesse de l'enveloppe de Snell}

Résumé : Nous analysons les propriètès de robustesse de l'équation de l'évolution backward de l'enveloppe de Snell pour le problème d'arrêt optimal au temps discet. Nous considèrons une série de schémas d'approximation, y compris les approximations de type cut-off, schémas de discrétisation d'Euler, des modèles d'interpolation, les modèles de quantification, et la méthode de BroadieGlasserman. Dans chaque situation, nous fournissons des estimations de convergence non-asymptotique, y compris les bornes d'erreur $\mathbb{L}_{p}$ et les inégalités de concentration exponentielle. Nous en déduisons de ces estimations à partir d'une seule propriété générale de robustesse générale de semigroupes enveloppe de Snell. En particulier, cette analyse nous permet de retrouver des résultats de convergence existants pour la méthode de quantification et d'améliorer significativement la vitesse de convergence obtenue pour l'estimateur de BroadieGlasserman.

Dans la deuxième partie de l'article, nous proposons une nouvelle approche en utilisant une approximation d'arbre généalogique du processus de Markov en termes de référence d'un modèle de type génétique neutre. Contrairement à Broadie-Glasserman Monte Carlo modèles, le coût de calcul de cette nouvelle approximation particulaire stochastique est linéaire du nombre de points échantillonnés. Certains résultats des simulations sont fournies et de confirmer l'intérêt de ce nouvel algorithme.

Mots-clés : enveloppe de Snell, arrêt optimal, évaluation de l'option américain, arbre génétique, modèle particulaire d'interaction 


\section{Introduction}

The calculation of optimal stopping time of random processes, based on a given optimality criteria, is one of the major problems in stochastic control and optimal stopping theory, and particularly in financial mathematics with American options pricing and hedging. The present paper is restricted to the case of discrete time optimal stopping problem corresponding in finance to the case of Bermudan options.

It is well known that the price of the Bermudan option giving the opportunity to exercise a payoff $f_{k}$ at discrete dates $k=0, \cdots, n$, can be calculated by a backward dynamic programming formula. This recursion consists in comparing at each time step $k$ the immediate payoff $f_{k}$ and the expectation of the future gain (or the so-called continuation value), which precisely involves the Markov transition $M_{k+1}$ of the underlying assets process $\left(X_{k}\right)$.

One first goal of this paper is to provide a simple framework to analyze in unison most of the numerical schemes currently used in practice to approximate the Snell envelope, which are precisely based on the approximation of the dynamic programming recursion. The idea is to analyze the related approximation error in terms of robustness properties of the Snell envelope with respect to (w.r.t.) the pair parameters $\left(f_{k}, M_{k}\right)$. Hence, we include in our analysis approximation schemes which are defined in terms of some approximate pairs of functions and transitions $\left(\widehat{f}_{k}, \widehat{M}_{k}\right)_{k}>0$. Then, we deduce from the robustness Lemma 2.1. stated in the preliminary Section 2, non asymptotic convergence theorems, including $\mathbb{L}_{p}$-mean error bounds and related exponential inequalities for the deviations of Monte Carlo type approximation models.

In Section 3, this approach allows us to derive non asymptotic error bounds for deterministic approximation schemes such as cut-off techniques, Euler type discrete time approximations, quantization tree models, interpolation type approximations, then recovering or improving some existing results or in some cases providing new bounds. We emphasize that this non asymptotic robustness analysis also allows to combine in a natural way several approximation models. For instance, under appropriate tightness conditions, cut-off techniques can be used to reduce the numerical analysis of the Snell envelope to compact state spaces and bounded functions $\widehat{f}_{n}$. In the same line of ideas, in designing any type of Monte Carlo approximation models, we can suppose that the transitions of the chain $X_{n}$ are known based on a preliminary analysis of Euler type approximation models.

In Section 4, we focus on two kind of Monte Carlo importance sampling approximation schemes. The first one is the Stochastic Mesh method introduced by M. Broadie and P. Glasserman in their seminal paper [5] (see also [22], for some recent refinements). The principle idea of this method is to operate a change of measure to replace conditional expectations by simple expectations involving the Markov transition densities w.r.t. some reference measures. The number of sampled points w.r.t. the reference measures $\eta_{n}$ required by this model can be constant in every exercise date. This technique avoids the explosion issue of the naive Monte Carlo method. As any full Monte Carlo type technique, the main advantage of their approach is that it applies to high dimensional Bermudan options with a finite possibly large, number of exercise dates. In [5], the authors provide a set of conditions under which the Monte Carlo importance scheme converges as the computational effort increases. However, the 
computing time grows quadratically with the number of sampled points in the stochastic mesh. In this context, in Section 4.2, we provide new non asymptotic estimates, including $\mathbb{L}_{p}$-mean error bounds and exponential concentration inequalities. Our analysis allows us to derive Theorem 4.7 improving significantly existing convergence results (see [5] or [1]).

The second type of Monte Carlo importance sampling scheme discussed in this article is another version of the Broadie-Glasserman model, called average density in the original article. The main advantage of this strategy comes from the fact that the sampling distribution $\eta_{n}$ can be chosen as the distribution of the random states $X_{n}$ of the reference Markov chain, even if the Radon Nikodym derivatives, $R_{n}(x, y)=\frac{d M_{n}(x, \bullet)}{d \eta_{n}}(y)$ is not known explicitly. We only assume that the Markov transitions $M_{n}(x,$.$) are absolutely continuous with respect to some$ measures $\lambda_{n}$. We can then approximate this function with empirical measure. In this situation, we can recover a similar approximation to the original stochastic mesh method, except that the Radon Nikodym derivatives, $R_{k+1}\left(\xi_{k}^{i}, \xi_{k+1}^{j}\right)$ is replaced by an approximation. The stochastic analysis of this particle model is provided in the second part of Section 4.2 and follows essentially the same line of arguments as the one of the Broadie-Glasserman model.

In the final part of the article, Section 5. we present a new Monte Carlo approach based on the genealogical tree evolution models associated with a neutral genetic model with mutation given by the Markov transitions $M_{n}$. The main advantage of this new strategy comes from the fact that the computational effort of the algorithm is now linear in the number of sampled points. We recall that a neutral genetic model is a Markov chain with a selection/mutation transition. During the mutation phase, the particles explore the state space independently according to the Markov transitions while the selection step induces interactions between the various particles. This type of model is frequently used in biology, and genetic algorithms literature (see for instance [14, and references therein).

An important observation concerns the genealogical tree structure of the genetic particle model that we consider. The main advantage of this path particle model comes from the fact that the occupation measure of the ancestral tree model converges in some sense to the distribution of the path of the reference Markov chain. It is also well known that the Snell envelope associated with a Markov chain evolving on some finite state space is easily computed using the tree structure of the chain evolution. Therefore, replacing the reference distribution $\mathbb{P}_{n}$ by its $N$-approximation $\mathbb{P}_{n}^{N}$, we define an $N$-approximated Markov model whose evolutions are described by the genealogical tree model defined above. We can then construct the approximation $\widehat{u}_{k}$ as the Snell envelope associated with this $N$-approximated Markov chain. Several estimates of convergence are provided in Section 5] Finally, some numerical simulations are performed and show the interest of our new algorithm.

\section{Preliminary}

In a discrete time setting, the problem is related to pricing of Bermuda options and is defined in terms of a given real valued stochastic process $\left(Z_{k}\right)_{0 \leq k \leq n}$, adapted to some increasing filtration $\mathcal{F}=\left(\mathcal{F}_{k}\right)_{0 \leq k \leq n}$ that represents the available information at any time $0 \leq k \leq n$. For any $k \in\{0, \ldots, n\}$, we let $\mathcal{T}_{k}$ be the set of all stopping times $\tau$ taking values in $\{k, \ldots, n\}$. The Snell envelope 
of $\left(Z_{k}\right)_{0 \leq k \leq n}$, is the stochastic process $\left(U_{k}\right)_{0 \leq k \leq n}$ defined for any $0 \leq k<n$ by the following backward equation

$$
U_{k}=Z_{k} \vee \mathbb{E}\left(U_{k+1} \mid \mathcal{F}_{k}\right),
$$

with the terminal condition $U_{n}=Z_{n}$. The main property of this stochastic process is that

$$
\begin{aligned}
U_{k} & =\sup _{\tau \in \mathcal{T}_{k}} \mathbb{E}\left(Z_{\tau} \mid \mathcal{F}_{k}\right)=\mathbb{E}\left(Z_{\tau_{k}^{*}} \mid \mathcal{F}_{k}\right) \\
\text { with } \quad \tau_{k}^{*} & =\min \left\{k \leq l \leq n: U_{l}=Z_{l}\right\} \in \mathcal{T}_{k} .
\end{aligned}
$$

At this level of generality, in the absence of any additional information on the sigma-fields $\mathcal{F}_{n}$, or on the terminal random variable $Z_{n}$, no numerical computation of the Snell envelop is available. To get one step further, we assume that $\left(\mathcal{F}_{n}\right)_{n \geq 0}$ is the natural filtration associated with some Markov chain $\left(X_{n}\right)_{n \geq 0}$ taking values in some sequence of measurable state spaces $\left(E_{n}, \mathcal{E}_{n}\right)_{n \geq 0}$. We let $\eta_{0}=\operatorname{Law}\left(X_{0}\right)$ be the initial distribution on $E_{0}$, and we denote by $M_{n}\left(x_{n-1}, d x_{n}\right)$ the elementary Markov transition of the chain from $E_{n-1}$ into $E_{n}$. We also assume that $Z_{n}=f_{n}\left(X_{n}\right)$, for some collection of nonnegative measurable functions $f_{n}$ on $E_{n}$. In this situation, the computation of the Snell envelope amounts to solve the following backward functional equation

$$
\begin{aligned}
u_{k} & =\mathcal{H}_{k+1}\left(u_{k+1}\right) \\
& =\max \left(f_{k}, M_{k+1}\left(u_{k+1}\right)\right)=f_{k} \vee M_{k+1}\left(u_{k+1}\right),
\end{aligned}
$$

for any $0 \leq k<n$, with the terminal value $u_{n}=f_{n}$. In the above displayed formula, $M_{k+1}\left(u_{k+1}\right)$ stands for the measurable function on $E_{k}$ defined for any $x_{k} \in E_{k}$ by the conditional expectation formula

$$
\begin{aligned}
M_{k+1}\left(u_{k+1}\right)\left(x_{k}\right) & =\int_{E_{k+1}} M_{k+1}\left(x_{k}, d x_{k+1}\right) u_{k+1}\left(x_{k+1}\right) \\
& =\mathbb{E}\left(u_{k+1}\left(X_{k+1}\right) \mid X_{k}=x_{k}\right)
\end{aligned}
$$

We let $\mathcal{H}_{k, l}=\mathcal{H}_{k+1} \circ \mathcal{H}_{k+1, l}$, with $k \leq l \leq n$, be the nonlinear semigroups associated with the backward equations (2.2). We use the convention $\mathcal{H}_{k, k}=I d$, the identity operator, so that $u_{k}=\mathcal{H}_{k, l}\left(u_{l}\right)$, for any $k \leq l \leq n$. Given a sequence of bounded integral operator $M_{k}$ from some state space $E_{k-1}$ into another $E_{k}$, let us denote by $M_{k, l}$ the composition operator such that $M_{k, l}:=$ $M_{k+1} M_{k+2} \cdots M_{l}$, for any $k \leq l$, with the convention $M_{k, k}=I d$, the identity operator. With this notation, one can check that a necessary and sufficient condition for the existence of the Snell envelope $\left(u_{k}\right)_{0 \leq k \leq n}$ is that $M_{k, l} f_{l}(x)<\infty$ for any $1 \leq k \leq l \leq n$, and any state $x \in E_{k}$. To check this claim, we simply notice that

$f_{k} \leq u_{k} \leq f_{k}+M_{k+1} u_{k+1}, \forall 1 \leq k \leq n \Longrightarrow f_{k} \leq u_{k} \leq \sum_{k \leq l \leq n} M_{k, l} f_{l}, \forall 1 \leq k \leq n$.

From the readily proved Lipschitz property $\left|\mathcal{H}_{k}(u)-\mathcal{H}_{k}(v)\right| \leq M_{k+1}(|u-v|)$, for any functions $u, v$ on $E_{k}$, we also have that

$$
\left|\mathcal{H}_{k, l}(u)-\mathcal{H}_{k, l}(v)\right| \leq M_{k, l}(|u-v|)
$$


for any functions $u, v$ on $E_{l}$, and any $k \leq l \leq n$.

Even if it looks innocent, the numerical solving of the recursion (2.2) often requires extensive calculations. The central problem is to compute the conditional expectations $M_{k+1}\left(u_{k+1}\right)$ on the whole state space $E_{k}$, at every time step $0 \leq k<n$. For Markov chain models taking values in some finite state spaces (with a reasonably large cardinality), the above expectations can be easily computed by a simple backward inspection of the whole realization tree that lists all possible outcomes and every transition of the chain. In more general situations, we need to resort to some approximation strategy. Most of the numerical approximation schemes amount to replacing the pair of functions and Markov transitions $\left(f_{k}, M_{k}\right)_{0 \leq k \leq n}$ by some approximation model $\left(\widehat{f}_{k}, \widehat{M}_{k}\right)_{0 \leq k \leq n}$ on some possibly reduced measurable subsets $\widehat{E}_{k} \subset E_{k}$. We let $\widehat{u}_{k}$ be the Snell envelope on $\widehat{E}_{k}$ associated with the functions $\widehat{f}_{k}$ and the sequence of integral operators $\widehat{M}_{k}$ from $\widehat{E}_{k-1}$ into $\widehat{E}_{k}$. As in (2.2), the computation of the Snell envelope $\widehat{u}_{k}$ amounts to solve the following backward functional equation

$$
\widehat{u}_{k}=\widehat{\mathcal{H}}_{k+1}\left(\widehat{u}_{k+1}\right)=\widehat{f}_{k} \vee \widehat{M}_{k+1}\left(\widehat{u}_{k+1}\right) \text {. }
$$

We let $\widehat{\mathcal{H}}_{k, l}=\widehat{\mathcal{H}}_{k+1} \circ \widehat{\mathcal{H}}_{k+1, l}$, with $k \leq l \leq n$, be the nonlinear semigroups associated with the backward equations (2.5), so that $\widehat{u}_{k}=\widehat{\mathcal{H}}_{k, l}\left(\widehat{u}_{l}\right)$, for any $k \leq l \leq n$. Using the elementary inequality $\left|\left(a \vee a^{\prime}\right)-\left(b \vee b^{\prime}\right)\right| \leq|a-b|+\left|a^{\prime}-b^{\prime}\right|$, which is valid for any $a, a^{\prime}, b, b^{\prime} \in \mathbb{R}$, for any $0 \leq k<n$ and for any functions $u$ on $E_{k+1}$ one readily obtains the local approximation inequality

$$
\left|\mathcal{H}_{k+1}(u)-\widehat{\mathcal{H}}_{k+1}(u)\right| \leq\left|f_{k}-\widehat{f}_{k}\right|+\left|\left(M_{k+1}-\widehat{M}_{k+1}\right)(u)\right|
$$

To transfer these local estimates to the semigroups $\mathcal{H}_{k, l}$ and $\widehat{\mathcal{H}}_{k, l}$ we use the same perturbation analysis as the one presented [10, 12, 21, 28] in the context of nonlinear filtering semigroups and particle approximation models. The difference between the approximate and the exact Snell envelope can be written as a telescoping sum

$$
u_{k}-\widehat{u}_{k}=\sum_{l=k}^{n}\left[\widehat{\mathcal{H}}_{k, l}\left(\mathcal{H}_{l+1}\left(u_{l+1}\right)\right)-\widehat{\mathcal{H}}_{k, l}\left(\widehat{\mathcal{H}}_{l+1}\left(u_{l+1}\right)\right)\right]
$$

setting for simplicity $\mathcal{H}_{n+1}\left(u_{n+1}\right)=u_{n}$ and $\widehat{\mathcal{H}}_{n+1}\left(u_{n+1}\right)=\widehat{u}_{n}$, for $l=n$. Combining the Lipschitz property (2.4) of the semigroup $\widehat{\mathcal{H}}_{k, l}$ with the local estimate (2.6), one finally gets the following robustness lemma, which is a natural and fundamental tool for the analysis of the Snell envelope approximations.

Lemma 2.1 For any $0 \leq k<n$, on the state space $\widehat{E}_{k}$, we have that

$$
\left|u_{k}-\widehat{u}_{k}\right| \leq \sum_{l=k}^{n} \widehat{M}_{k, l}\left|f_{l}-\widehat{f}_{l}\right|+\sum_{l=k}^{n-1} \widehat{M}_{k, l}\left|\left(M_{l+1}-\widehat{M}_{l+1}\right) u_{l+1}\right|
$$

The perturbation analysis of nonlinear semigroups described above and the resulting robustness lemma are not really new. As we mentioned above, it is a rather standard tool in approximation theory and numerical probability. More precisely, these Lipschitz type estimates are often used by induction or as 
an intermediate technical step in the proof of a convergence theorem of some particular approximation scheme.

In the context of optimal stopping problems and numerical quantization schemes, these techniques are used for instance in the papers of Egloff [16] and Gobet, Lemor and Warin [19] or Pagès [24]. To the best of our knowledge, the general and abstract formulation given above and its direct application to different approximation models seems to be the first result of this type for this class of models.

Besides the fact that the convergence of many Snell approximation schemes result from a single robustness property, the lemma 2.1 can be used sequentially and without further work to obtain non asymptotic estimates for models combining several levels of approximations. In the same vein, and whenever it is possible, lemma 2.1 can also be used as a technical tool to reduce the analysis of Snell approximation models on compact state spaces or even on finite but possibly large quantization trees or Monte Carlo type grids.

To interpret better the $\mathbb{L}_{p}$-mean error bounds appearing in this article, we end this section with the following lemma.

Lemma 2.2 Suppose the estimates have the following form:

$$
\sqrt{N} \sup _{x \in E_{k}} \mathbb{E}\left(\left|u_{k}(x)-\widehat{u}_{k}(x)\right|^{p}\right)^{\frac{1}{p}} \leq a(p) b_{k}(n),
$$

where $b_{k}(n)$ are some finite constants whose values do not depend on the parameter $p$ and $a(p)$ is a collection of constants such that for all nonnegative integer $r$ :

$$
a(2 r)^{2 r}=(2 r)_{r} 2^{-r} \quad \text { and } \quad a(2 r+1)^{2 r+1}=\frac{(2 r+1)_{r+1}}{\sqrt{r+1 / 2}} 2^{-(r+1 / 2)},
$$

with the notation $(q)_{p}=q ! /(q-p)$ !, for any $1 \leq p \leq q$. Then we deduce the following exponential concentration inequality

$$
\sup _{x \in E_{k}} \mathbb{P}\left(\left|u_{k}\left(x_{k}\right)-\widehat{u}_{k}\left(x_{k}\right)\right|>\frac{b_{k}(n)}{\sqrt{N}}+\epsilon\right) \leq \exp \left(-N \epsilon^{2} /\left(2 b_{k}(n)^{2}\right)\right) .
$$

\section{Proof:}

This result is a direct consequence from the fact that, for any nonnegative random variable $U$, if there exists a bounded positive real $b$ such that

$$
\forall r \geq 1 \quad \mathbb{E}\left(U^{r}\right)^{\frac{1}{r}} \leq a(r) b
$$

where $a(r)$ is defined by (2.7), then

$$
\mathbb{P}(U \geq b+\epsilon) \leq \exp \left(-\epsilon^{2} /\left(2 b^{2}\right)\right) .
$$

To check this implication, we first notice that

$$
\mathbb{P}(U \geq b+\epsilon) \leq \inf _{t \geq 0}\left\{e^{-t(b+\epsilon)} \mathbb{E}\left[e^{t U}\right]\right\} .
$$

Then developing the exponential and using the moments boundedness assumption implies that for all $t \geq 0$

$$
\mathbb{E}\left(e^{t U}\right) \leq \exp \left(\frac{(b t)^{2}}{2}+b t\right)
$$


Finally

$$
\mathbb{P}(U \geq b+\epsilon) \leq \exp \left(-\sup _{t \geq 0}\left(\epsilon t-\frac{(b t)^{2}}{2}\right)\right)
$$

Hence, for any approximation model whose $\mathbb{L}_{p}$-mean error bound has the form listed in the above lemma, we can interpret that the probability that the approximation model makes some level of error is exponentially small.

\section{Some deterministic approximation models}

In this section, we analyze the robustness of the Snell envelope w.r.t. some deterministic approximation schemes that are parts of many algorithms proposed to approximate the Snell envelope. Hence, the non asymptotic error bounds provided in this section can be applied and combined to derive convergence rates for such algorithms. We recover or improve previous results and in some cases, state new error bounds.

\subsection{Cut-off type models}

It is often useful, when computing the Snell envelope, to approximate the state space by a compact set. Indeed, Glasserman and Yu (2004) [18] showed that for standard (unbounded) models (like Black-Scholes), the Monte Carlo estimation requires samples of exponential size in the number of variables of the value function, whereas the bounded state space assumption enables to estimate the Snell envelope from samples of polynomial size in the number of variables. For instance, in [17, the authors propose a new algorithm that first requires a cut off step which consists of replacing the price process by another process killed at first exit from a given bounded set. However, no bound is provided for the error induced by this cut off approximation. In this section, we formalize a general cut-off model and provide some bounds on the error induced on the Snell envelope.

We suppose that $E_{n}$ are topological spaces with $\sigma$-fields $\mathcal{E}_{n}$ that contain the Borel $\sigma$-field on $E_{n}$. Our next objective is to find conditions under which we can reduce the backward functional equation (2.2) to a sequence of compact sets $\widehat{E}_{n}$.

To this end, we further assume that the initial measure $\eta_{0}$ and the Markov transition $M_{n}$ of the chain $X_{n}$ satisfy the following tightness property: For every sequence of positive numbers $\epsilon_{n} \in[0,1[$, there exists a collection of compact subsets $\widehat{E}_{n} \subset E_{n}$ s.t.

$$
\eta_{0}\left(\widehat{E}_{0}^{c}\right) \leq \epsilon_{0} \quad \text { and } \quad \forall n \geq 0 \quad \sup _{x_{n} \in \widehat{E}_{n}} M_{n+1}\left(x_{n}, \widehat{E}_{n+1}^{c}\right) \leq \epsilon_{n+1}
$$

For instance, this condition is clearly met for regular Gaussian type transitions on the Euclidean space, for some collection of increasing compact balls.

In this situation, a natural cut off consists in considering the Markov transitions $\widehat{M}_{k}$ restricted to the compact sets $\widehat{E}_{k}$

$$
\forall x \in \widehat{E}_{k-1} \quad \widehat{M}_{k}(x, d y):=\frac{M_{k}(x, d y) 1_{\widehat{E}_{k}}}{M_{k}\left(1_{\widehat{E}_{k}}\right)(x)} .
$$


These transitions are well defined as soon as $M_{k}\left(x, \widehat{E}_{k}\right)>0$, for any $x \in \widehat{E}_{k-1}$. Using the decomposition

$$
\begin{aligned}
{\left[\widehat{M}_{k}-M_{k}\right]\left(u_{k}\right) } & =\widehat{M}_{k}\left(u_{k}\right)-M_{k}\left(1_{\widehat{E}_{k}} u_{k}\right)-M_{k}\left(1_{\widehat{E}_{k}^{c}} u_{k}\right) \\
& =\left(1-\frac{1}{M_{k}\left(1_{\widehat{E}_{k}}\right)}\right) M_{k}\left(u_{k} 1_{\widehat{E}_{k}}\right)-M_{k}\left(1_{\widehat{E}_{k}^{c}} u_{k}\right) \\
& =\frac{M_{k}\left(1_{\widehat{E}_{k}^{c}}\right)}{M_{k}\left(1_{\widehat{E}_{k}}\right)} M_{k}\left(u_{k} 1_{\widehat{E}_{k}}\right)-M_{k}\left(1_{\widehat{E}_{k}^{c}} u_{k}\right) .
\end{aligned}
$$

Then using Lemma 2.1 yields

$$
\begin{aligned}
\left\|u_{k}-\widehat{u}_{k}\right\|_{\widehat{E}_{k}} & :=\sup _{x \in \widehat{E}_{k}}\left|u_{k}(x)-\widehat{u}_{k}(x)\right| \\
& \leq \sum_{l=k+1}^{n}\left[\left\|\frac{M_{l}\left(1_{\widehat{E}_{l}^{c}}\right)}{M_{l}\left(1_{\widehat{E}_{l}}\right)}\right\|_{\widehat{E}_{l-1}}\left\|M_{l}\left(u_{l} 1_{\widehat{E}_{l}}\right)\right\|_{\widehat{E}_{l-1}}+\left\|M_{l}\left(u_{l} 1_{\widehat{E}_{l}^{c}}\right)\right\|_{\widehat{E}_{l-1}}\right] \\
& \leq \sum_{l=k+1}^{n}\left[\frac{\epsilon_{l}}{1-\epsilon_{l}}\left\|M_{l}\left(u_{l}\right)\right\|_{\widehat{E}_{l-1}}+\left\|M_{l}\left(u_{l}^{2}\right)\right\|_{\widehat{E}_{l-1}}^{1 / 2} \epsilon_{l}^{1 / 2}\right] .
\end{aligned}
$$

We summarize the above discussion with the following result.

Theorem 3.1 We assume that the tightness condition $(\mathcal{T})$ is met, for every sequence of positive numbers $\epsilon_{n} \in[0,1[$, and for some collection of compact subsets $\widehat{E}_{n} \subset E_{n}$. In this situation, for any $0 \leq k \leq n$, we have that

$$
\left\|u_{k}-\widehat{u}_{k}\right\|_{\widehat{E}_{k}} \leq \sum_{l=k+1}^{n} \frac{\epsilon_{l}^{1 / 2}}{1-\epsilon_{l}^{1 / 2}}\left\|M_{l}\left(u_{l}^{2}\right)\right\|_{\widehat{E}_{l-1}}^{1 / 2} .
$$

We notice that

$$
u_{k} \leq \sum_{l=k}^{n} M_{k, l}\left(f_{l}\right)
$$

and therefore

$$
\left\|M_{k}\left(u_{k}^{2}\right)\right\|_{\widehat{E}_{k-1}} \leq(n-k+1) \sum_{l=k}^{n}\left\|M_{k-1, l}\left(f_{l}\right)^{2}\right\|_{\widehat{E}_{k-1}} .
$$

Consequently, one can find sets $\left(\widehat{E}_{l}\right)_{k<l \leq n}$ so that $\left\|u_{k}-\widehat{u}_{k}\right\|_{\widehat{E}_{k}}$ is as small as one wants as soon as $\left\|M_{k, l}\left(f_{l}\right)^{2}\right\|_{\widehat{E}_{k}}<\infty$, for any $0 \leq k<l \leq n$. A similar cut-off approach was intoduced and analyzed in Bouchard and Touzi [6], but the cut-off was operated on some regression functions and not on the transition kernels.

\subsection{Euler approximation models}

In several application model areas, the discrete time Markov chain $\left(X_{k}\right)_{k \geq 0}$ is often given in terms of an $\mathbb{R}^{d}$-valued and continuous time process $\left(X_{t}\right)_{t \geq 0}$ given by a stochastic differential equation of the following form

$$
d X_{t}=a\left(X_{t}\right) d t+b\left(X_{t}\right) d W_{t}, \quad \operatorname{law}\left(X_{0}\right)=\eta_{0},
$$


where $\eta_{0}$ is a known distribution on $\mathbb{R}^{d}$, and $a, b$ are known functions, and $W$ is a $d$-dimensional Wiener process. Except in some particular instances, the time homogeneous Markov transitions $M_{k}=M$ are usually unknown, and we need to resort to an Euler approximation scheme.

In this situation, any approximation or the Snell envelope, which is based on simulations of the price process will be impacted by the error induced by the Euler scheme used in simulations. We propose here to provide bounds for this error. Notice that in this setting, the exercise dates are discrete and fixed, so that our results are not comparable with those from Dupuis and Wang (2004) [15] who analyzed the convergence of the discrete time optimal stopping problem to a continuous time optimal stopping when the frequency of exercise dates increases to infinity. Similarly, for numerical approximations of Backward Stochastic Differential Equations (BSDE), [6] and [19] also analysed the case where the number exercise opportunities grows to infinity.

The discrete time approximation model with a fixed time step $1 / m$ is defined by the following recursive formula

$$
\begin{aligned}
\widehat{\xi}_{0}(x) & =x \\
\widehat{\xi}_{\frac{(i+1)}{m}}(x) & =\widehat{\xi}_{\frac{i}{m}}(x)+a\left(\widehat{\xi}_{\frac{i}{m}}(x)\right) \frac{1}{m}+b\left(\widehat{\xi}_{\frac{i}{m}}(x)\right) \frac{1}{\sqrt{m}} \epsilon_{i} .
\end{aligned}
$$

where the $\epsilon_{i}$ 's are i.i.d. centered and $\mathbb{R}^{d}$-valued Gaussian vectors with unit covariance matrix. The chain $\left(\widehat{\xi}_{k}\right)_{k \geq 0}$ is an homogeneous Markov with a transition kernel which we denote by $\widehat{M}$.

We further assume that the functions $a$ and $b$ are twice differentiable, with bounded partial derivatives of orders 1 and 2 , and the matrix $\left(b b^{*}\right)(x)$ is uniformly non-degenerate.

In this situation, the integral operators $M$ and $\widehat{M}$ admit densities, denoted by $p$ and $\widehat{p}$. According to Bally and Talay [4, we have that

$$
[p \vee \widehat{p}] \leq c q \text { and } m|\widehat{p}-p| \leq c q
$$

with the Gaussian density $q\left(x, x^{\prime}\right):=\frac{1}{\sqrt{2 \pi} \sigma} e^{-\frac{1}{2 \sigma^{2}}\left|x-x^{\prime}\right|^{2}}$, and a pair of constants $(c, \sigma)$ depending only on the pair of functions $(a, b)$. Let $Q$, be the Markov integral operator on $\mathbb{R}^{d}$ with density $q$. We consider a sequence of functions $\left(f_{k}\right)_{0 \leq k \leq n}$ on $\mathbb{R}^{d}$. We let $\left(u_{k}\right)_{0 \leq k \leq n}$ and $\left(\widehat{u}_{k}\right)_{0 \leq k \leq n}$ be the Snell envelopes on $\mathbb{R}^{d}$ associated to the pair $\left(M, f_{k}\right)$ and $\left(\widehat{M}, f_{k}\right)$. Using Lemma 2.1, we readily obtain the following estimate

$$
\left|u_{k}-\widehat{u}_{k}\right| \leq \sum_{l=k}^{n-1} \widehat{M}^{l-k}\left|(M-\widehat{M}) u_{l+1}\right| \leq \frac{c}{m} \sum_{l=k}^{n-1} \widehat{M}^{l-k} Q\left|u_{l+1}\right|
$$

Rather crude upper bounds that do not depend on the approximation kernels $\widehat{M}$ can be derived using the first inequality in (3.2)

$$
\left|u_{k}-\widehat{u}_{k}\right| \leq \frac{1}{m} \sum_{l=1}^{n-k} c^{l} Q^{l}\left|u_{l+k}\right| .
$$


Recalling that $u_{l+k} \leq \sum_{l+k \leq l^{\prime} \leq n} M^{l^{\prime}-(l+k)} f_{l^{\prime}}$, we also have that

$$
\begin{aligned}
\left|u_{k}-\widehat{u}_{k}\right| & \leq \frac{1}{m} \sum_{l=1}^{n-k} c^{l} Q^{l} \sum_{l+k \leq l^{\prime} \leq n} c^{l^{\prime}-(l+k)} Q^{l^{\prime}-(l+k)} f_{l^{\prime}} \\
& \leq \frac{1}{m} \sum_{l=1}^{n-k} \sum_{l+k \leq l^{\prime} \leq n} c^{l^{\prime}-k} Q^{l^{\prime}-k} f_{l^{\prime}}=\frac{1}{m} \sum_{1 \leq l \leq n-k} l c^{l} Q^{l} f_{k+l} .
\end{aligned}
$$

We summarize the above discussion with the following theorem.

Theorem 3.2 Suppose the functions $\left(f_{k}\right)_{0 \leq k \leq n}$ on $\mathbb{R}^{d}$ are chosen such that $Q^{l} f_{k+l}(x)<\infty$, for any $x \in \mathbb{R}^{d}$, and $1 \leq k+l \leq n$. Then, for any $0 \leq l \leq n$, we have the inequalities

$$
\left|u_{k}-\widehat{u}_{k}\right| \leq \frac{c}{m} \sum_{l=k}^{n-1} \widehat{M}^{l-k} Q\left|u_{l+1}\right| \leq \frac{1}{m} \sum_{1 \leq l \leq n-k} l c^{l} Q^{l} f_{k+l} .
$$

\subsection{Interpolation type models}

Most algorithms proposed to approximate the Snell envelope provide discrete approximations $\hat{u}_{k}^{i}$ at some discrete (potentially random) points $\xi_{k}^{i}$ of $E_{k}$. However, for several purposes, it can be interesting to consider approximations $\hat{u}_{k}$ of functions $u_{k}$ on the whole space $E_{k}$. One motivation to do so is, for instance, to be able to define a new (low biased) estimator, $\bar{U}_{k}$, using a Monte Carlo approximation of (2.1), with a stopping rule $\hat{\tau}_{k}$ associated with the approximate Snell envelope $\hat{u}_{k}$, by replacing $u_{k}$ by $\hat{u}_{k}$ in the characterization of the optimal stopping time $\tau_{k}^{*}(2.1)$, i.e.

$$
\bar{U}_{k}=\frac{1}{M} \sum_{i=1}^{M} f_{\hat{\tau}_{k}^{i}}\left(X_{\hat{\tau}_{k}^{i}}^{i}\right) \quad \text { with } \quad \hat{\tau}_{k}^{i}=\min \left\{k \leq l \leq n: \hat{u}_{l}\left(X_{l}^{i}\right)=f_{l}\left(X_{l}^{i}\right)\right\} .
$$

where $X^{i}=\left(X_{1}^{i}, \cdots, X_{n}^{i}\right)$ are i.i.d. path according to the reference Markov chain dynamic.

In this section, we analyze non asymptotic errors of some specific approximation schemes providing such interpolated estimators $\hat{u}_{k}$ of $u_{k}$ on the whole state $E_{k}$. Let $\widehat{M}_{k+1}=\mathcal{I}_{k} \widetilde{M}_{k+1}$ be the composition of the Markov transition $\widetilde{M}_{k+1}$ from a finite set $S_{k}$ into the whole state space $E_{k+1}$, with an auxiliary interpolation type and Markov operator $\mathcal{I}_{k}$ from $E_{k}$ into $S_{k}$, so that

$$
\forall x_{k} \in S_{k} \quad \mathcal{I}_{k}\left(x_{k}, d s\right)=\delta_{x_{k}}(d s),
$$

and such that the integrals

$$
x \in E_{k} \mapsto \mathcal{I}_{k}\left(\varphi_{k}\right)(x)=\int_{S_{k}} \mathcal{I}_{k}(x, d s) \varphi_{k}(s),
$$

of any function $\varphi_{k}$ on $S_{k}$ are easily computed starting from any point $x_{k}$ in $E_{k}$. We further assume that the finite state spaces $S_{k}$ are chosen so that

$$
\left\|f-\mathcal{I}_{k} f\right\|_{E_{k}} \leq \epsilon_{k}\left(f,\left|S_{k}\right|\right) \rightarrow 0 \quad \text { as } \quad\left|S_{k}\right| \rightarrow \infty,
$$


for continuous functions $f_{k}$ on $E_{k}$. An example of interpolation transition $\mathcal{I}_{k}$ is provided hereafter. We let $\widehat{M}_{k}=\mathcal{I}_{k-1} \widetilde{M}_{k}$ be the composition operator on the state spaces $\widehat{E}_{k}=E_{k}$.

The approximation models $\widetilde{M}_{k}$ are non necessarily deterministic. In [13], we examined the situation where

$$
\forall s \in S_{k} \quad \widetilde{M}_{k}(s, d x)=\frac{1}{N_{k}} \sum_{1 \leq i \leq N_{k}} \delta_{X_{k}^{i}(s)}(d x),
$$

where $X_{k}^{i}(s)$ stands for a collection of $N_{k}$ independent random variables with common law $M_{k}(s, d x)$.

Theorem 3.3 We suppose that the Markov transitions $M_{k}$ are Feller, in the sense that $M_{k}\left(C\left(E_{k}\right)\right) \subset C\left(E_{k-1}\right)$, where $C\left(E_{k}\right)$ stands for the space of continuous functions on the $E_{k}$. We let $\left(u_{k}\right)_{0 \leq k \leq n}$, and respectively $\left(\widehat{u}_{k}\right)_{0 \leq k \leq n}$ be the Snell envelope associated with the functions $f_{k}=\widehat{f}_{k}$, and the Markov transitions $M_{k}$, and respectively $\widehat{M}_{k}=\mathcal{I}_{k-1} \widehat{M}_{k}$ on the state spaces $\widehat{E}_{k}=E_{k}$.

$$
\left\|u_{k}-\widehat{u}_{k}\right\|_{E_{k}} \leq \sum_{l=k}^{n-1}\left[\epsilon_{l}\left(M_{l+1} u_{l+1},\left|S_{l}\right|\right)+\left\|\left(M_{l+1}-\widetilde{M}_{l+1}\right) u_{l+1}\right\|_{S_{l}}\right] .
$$

The proof of the theorem is a direct consequence of Lemma 2.1 combined with the following decomposition

$$
\begin{aligned}
& \left\|u_{k}-\widehat{u}_{k}\right\|_{E_{k}} \\
& \left.\quad \leq \sum_{l=k}^{n-1}\left[\|\left(I d-\mathcal{I}_{l}\right) M_{l+1}\right) u_{l+1}\left\|_{E_{l}}+\right\| \mathcal{I}_{l}\left(M_{l+1}-\widetilde{M}_{l+1}\right) u_{l+1} \|_{E_{l}}\right]
\end{aligned}
$$

We illustrate these results in the typical situation where the space $E_{k}$ are the convex hull generated by the finite sets $S_{k}$. Firstly, we present the definition of the interpolation operators. We let $\mathcal{P}=\left\{\mathcal{P}^{1}, \ldots, \mathcal{P}^{m}\right\}$ be a partition of a convex and compact space $E$ into simplexes with disjoint non empty interiors, so that $E=\cup_{1 \leq i \leq m} \mathcal{P}_{i}$. We denote by $\delta(\mathcal{P})$ the refinement degree of the partition $\mathcal{P}$

$$
\delta(\mathcal{P}):=\sup _{1 \leq i \leq m} \sup _{x, y \in \mathcal{P}_{i}}\|x-y\| .
$$

We let $S=\mathcal{V}(\mathcal{P})$ be the set of vertices of these simplexes. We denote by $\mathcal{I}$ be the interpolation operator defined by $\mathcal{I}(f)(s)=f(s)$, if $s \in S$, and if $x$ belongs to some simplex $\mathcal{P}^{j}$ with vertices $\left\{x_{1}^{j}, \ldots, x_{d_{j}}^{j}\right\}$

$$
\mathcal{I}(f)\left(\sum_{1 \leq i \leq d_{j}} \lambda_{i} x_{j}^{i}\right)=\sum_{1 \leq i \leq d_{j}} \lambda_{i} f\left(x_{i}^{j}\right)
$$

where the barycenters $\left(\lambda_{i}\right)_{1 \leq i \leq d_{j}}$ are the unique solution of

$$
x=\sum_{1 \leq i \leq d_{j}} \lambda_{i} x_{i}^{j} \quad \text { with } \quad\left(\lambda_{i}\right)_{1 \leq i \leq d_{j}} \in[0,1]^{d_{j}} \quad \text { and } \quad \sum_{1 \leq i \leq d_{j}} \lambda_{i}=1
$$

The Markovian interpretation is that starting from $x$, one choses the " closest simplex" and then one chooses one of its vertices $x_{i}$ with probability $\lambda_{i}$. 
For any $\delta>0$, we let $\omega(f, \delta)$ be the $\delta$-modulus of continuity of a function $f \in C(E)$

$$
\omega(f, \delta):=\sup _{(x, y) \in E:\|x-y\| \leq \delta}|f(x)-f(y)| .
$$

The following technical Lemma provides a simple way to check condition (3.4) for interpolation kernels.

Lemma 3.4 Then for any $f, g \in C(E)$,

$$
\sup _{x \in E}|f(x)-\mathcal{I} g(x)| \leq \max _{x \in S}|f(x)-g(x)|+\omega(f, \delta(\mathcal{P}))+\omega(g, \delta(\mathcal{P})) .
$$

In particular, we have that

$$
\sup _{x \in E}|f(x)-\mathcal{I} f(x)| \leq \omega(f, \delta(\mathcal{P})) .
$$

Proof:

Suppose $x$ belongs to some simplex $\mathcal{P}^{j}$ with vertices $\left\{x_{1}^{j}, \ldots, x_{d_{j}}^{j}\right\}$, and let $\left(\lambda_{i}\right)_{1 \leq i \leq d_{j}}$ be the barycenter parameters $x=\sum_{1 \leq i \leq d_{j}} \lambda_{i} x_{j}^{i}$. Since we have $\mathcal{I} g\left(x_{i}^{j}\right)=g\left(x_{i}^{j}\right)$, and $\mathcal{I} g\left(x_{i}^{j}\right)=g\left(x_{i}^{j}\right)$ for any $i \in\left\{1, \ldots, d_{j}\right\}$, it follows that

$$
\begin{aligned}
|f(x)-\mathcal{I} g(x)| \leq & \sum_{i=1}^{d_{j}} \lambda_{i} \mid\left(f(x)-f\left(x_{i}^{j}\right)\left|+\sum_{i=1}^{d_{j}} \lambda_{i}\right| f\left(x_{i}^{j}\right)-\mathcal{I} g\left(x_{i}^{j}\right) \mid\right. \\
& +\sum_{i=1}^{d_{j}} \lambda_{i}\left|\mathcal{I} g\left(x_{i}^{j}\right)-g(x)\right| \\
= & \sum_{i=1}^{d_{j}} \lambda_{i} \mid\left(f(x)-f\left(x_{i}^{j}\right)\left|+\sum_{i=1}^{d_{j}} \lambda_{i}\right| f\left(x_{i}^{j}\right)-g\left(x_{i}^{j}\right) \mid\right. \\
& +\sum_{i=1}^{d_{j}} \lambda_{i}\left|g\left(x_{i}^{j}\right)-g(x)\right| .
\end{aligned}
$$

This implies that

$$
\sup _{x \in \mathcal{P}^{j}}|f(x)-\mathcal{I} g(x)| \leq \max _{x \in \mathcal{P}^{j}}|f(x)-g(x)|+\omega\left(f, \delta\left(\mathcal{P}^{j}\right)\right)+\omega\left(g, \delta\left(\mathcal{P}^{j}\right)\right),
$$

with

$$
\omega\left(f, \delta\left(\mathcal{P}^{j}\right)\right)=\sup _{\|x-y\| \leq \delta\left(\mathcal{P}^{j}\right)}|f(x)-f(y)| \quad \text { and } \quad \delta\left(\mathcal{P}^{j}\right):=\sup _{x, y \in \mathcal{P}^{j}}\|x-y\| .
$$

The end of the proof is now clear.

Combining (3.5) and (3.6), we obtain the following result.

Proposition 3.5 We let $\mathcal{P}_{k}=\left\{\mathcal{P}_{k}^{1}, \ldots, \mathcal{P}_{k}^{m_{k}}\right\}$ be a partition of a convex and compact space $E_{k}$ into simplexes with disjoint non empty interiors, so that $E_{k}=$ $\cup_{1 \leq i \leq m_{k}} \mathcal{P}_{i}$. We let $S_{k}=\mathcal{V}\left(\mathcal{P}_{k}\right)$ be the set of vertices of these simplexes. We let $\left(\widehat{u}_{k}\right)_{0 \leq k \leq n}$, be the Snell envelope associated with the functions $\widehat{f}_{k}=f_{k}$ and the Markov transitions $\widehat{M}_{k}=\mathcal{I}_{k-1} \widetilde{M}_{k}$ on the state spaces $E_{k}=\widehat{E}_{k}$.

$$
\left\|u_{k}-\widehat{u}_{k}\right\|_{E_{k}} \leq \sum_{l=k}^{n-1}\left[\omega\left(M_{l+1} u_{l+1}, \delta\left(\mathcal{P}_{l}\right)\right)+\left\|\left(M_{l+1}-\widetilde{M}_{l+1}\right) u_{l+1}\right\|_{S_{l}}\right] .
$$




\subsection{Quantization tree models}

Quantization tree models belong to the class of deterministic grid approximation methods. The basic idea consists in choosing finite space grids

$$
\widehat{E}_{k}=\left\{x_{k}^{1}, \ldots, x_{k}^{m_{k}}\right\} \subset E_{k}=\mathbb{R}^{d},
$$

and some neighborhoods measurable partitions $\left(A_{k}^{i}\right)_{1 \leq k \leq m_{k}}$ of the whole space $E_{k}$ such that the random state variable $X_{k}$ is suitably approximated, as $m_{k} \rightarrow$ $\infty$, by discrete random variables of the following form

$$
\widehat{X}_{k}:=\sum_{1 \leq i \leq m_{k}} x_{k}^{i} 1_{A_{k}^{i}}\left(X_{k}\right) \simeq X_{k} .
$$

The numerical efficiency of these quantization methods heavily depends on the choice of these grids. There exists various criteria to choose judiciously these objects, including minimal $\mathbb{L}_{p}$-quantization errors, that ensure that the corresponding Voronoi type quantized variable $\widehat{X}_{k}$ minimizes the $\mathbb{L}_{p}$ distance to the real state variable $X_{k}$. For further details on this subject, we refer the interested reader to the pioneering article of G. Pagès [24], and the series of articles of V. Bally, G. Pagès, and J. Printemps [2], G. Pagès and J. Printems [25], as well as G. Pagès , H. Pham and J. Printems [26], and references therein. The second approximation step of these quantization model consists in defining the coupled distribution of any pair of variables $\left(\widehat{X}_{k-1}, \widehat{X}_{k}\right)$ by setting

$$
\mathbb{P}\left(\widehat{X}_{k}=x_{k}^{j}, \widehat{X}_{k-1}=x_{k-1}^{i}\right)=\mathbb{P}\left(X_{k} \in A_{k}^{j}, X_{k-1} \in A_{k-1}^{i}\right),
$$

for any $1 \leq i \leq m_{k-1}$, and $1 \leq j \leq m_{k}$. This allows to interpret the quantized variables $\left(\widehat{X}_{k}\right)_{0 \leq k \leq n}$ as a Markov chain taking values in the states spaces $\left(\widehat{E}_{k}\right)_{0 \leq k \leq n}$ with Markov transitions

$$
\widehat{M}_{k}\left(x_{k-1}^{i}, x_{k}^{j}\right):=\mathbb{P}\left(\widehat{X}_{k}=x_{k}^{j} \mid \widehat{X}_{k-1}=x_{k-1}^{i}\right)=\mathbb{P}\left(X_{k} \in A_{k}^{j} \mid X_{k} \in A_{k-1}^{i}\right) \text {. }
$$

Using the decompositions

$$
\begin{aligned}
M_{k}(f)\left(x_{k-1}^{i}\right)= & \sum_{j=1}^{m_{k}} \int_{A_{k}^{j}} f(y) \mathbb{P}\left(X_{k} \in d y \mid X_{k-1}=x_{k-1}^{i}\right) \\
= & \sum_{j=1}^{m_{k}} \int_{A_{k}^{j}} f(y) \mathbb{P}\left(X_{k} \in d y \mid X_{k-1} \in A_{k-1}^{i}\right) \\
& +\int\left[M(f)\left(x_{k-1}^{i}\right)-M(f)(x)\right] \mathbb{P}\left(X_{k-1} \in d x \mid X_{k-1} \in A_{k-1}^{i}\right),
\end{aligned}
$$

and

$$
\widehat{M}_{k}(f)\left(x_{k-1}^{i}\right)=\sum_{j=1}^{m_{k}} \int_{A_{k}^{j}} f\left(x_{k}^{j}\right) \mathbb{P}\left(X_{k} \in d y \mid X_{k-1} \in A_{k-1}^{i}\right),
$$

we find that

$$
\begin{aligned}
& {\left[M_{k}-\widehat{M}_{k}\right](f)\left(x_{k-1}^{i}\right)} \\
& =\sum_{j=1}^{m_{k}} \int_{A_{k}^{j}}\left[f(y)-f\left(x_{k}^{j}\right)\right] \mathbb{P}\left(X_{k} \in d y \mid X_{k-1} \in A_{k-1}^{i}\right) \\
& +\int_{7303}^{j}\left[M(f)\left(x_{k-1}^{i}\right)-M(f)(x)\right] \mathbb{P}\left(X_{k-1} \in d x \mid X_{k-1} \in A_{k-1}^{i}\right) .
\end{aligned}
$$


We let $\operatorname{Lip}\left(\mathbb{R}^{d}\right)$ be the set of all Lipschitz functions $f$ on $\mathbb{R}^{d}$, and we set

$$
L(f)=\sup _{x, y \in \mathbb{R}^{d}, x \neq y} \frac{|f(x)-f(y)|}{|x-y|},
$$

for any $f \in \operatorname{Lip}\left(\mathbb{R}^{d}\right)$. We further assume that $M_{k}\left(\operatorname{Lip}\left(\mathbb{R}^{d}\right)\right) \subset \operatorname{Lip}\left(\mathbb{R}^{d}\right)$. From previous considerations, we find that

$$
\begin{aligned}
\left|\left[M_{k}-\widehat{M}_{k}\right](f)\left(x_{k-1}^{i}\right)\right| \leq & \left.L(f) \mathbb{E}\left[\left|X_{k}-\widehat{X}_{k}\right|^{p} \mid \widehat{X}_{k-1}=x_{k-1}^{i}\right)\right]^{\frac{1}{p}} \\
& +L\left(M_{k}(f)\right) \mathbb{E}\left(\left|X_{k-1}-\widehat{X}_{k-1}\right|^{p} \mid \widehat{X}_{k-1}=x_{k-1}^{i}\right)^{\frac{1}{p}} .
\end{aligned}
$$

This clearly implies that

$$
\begin{aligned}
\widehat{M}_{k, l}\left|\left(M_{l+1}-\widehat{M}_{l+1}\right) f\right|\left(x_{k}^{i}\right) \leq & L(f)\left[\mathbb{E}\left(\left|X_{l+1}-\widehat{X}_{l+1}\right|^{p} \mid \widehat{X}_{k}=x_{k}^{i}\right)\right]^{\frac{1}{p}} \\
& +L\left(M_{l+1}(f)\right) \mathbb{E}\left(\left|X_{l}-\widehat{X}_{l}\right|^{p} \mid \widehat{X}_{k}=x_{k}^{i}\right)^{\frac{1}{p}}
\end{aligned}
$$

We also observe that

$$
\begin{gathered}
\left(f_{k} \quad \text { and } \quad u_{k+1} \in \operatorname{Lip}\left(\mathbb{R}^{d}\right)\right) \\
\Downarrow \\
\left(u_{k} \in \operatorname{Lip}\left(\mathbb{R}^{d}\right) \quad \text { with } \quad L\left(u_{k}\right) \leq L\left(f_{k}\right) \vee L\left(M_{k+1}\left(u_{k+1}\right)\right)\right)
\end{gathered}
$$

Using Lemma 2.1] we readily arrive at the following Proposition similar to Theorem 2 in [2].

Proposition 3.6 Assume that $\left(f_{k}\right)_{0 \leq k \leq n} \in \operatorname{Lip}\left(\mathbb{R}^{d}\right)^{n+1}$, and $M_{k}\left(\operatorname{Lip}\left(\mathbb{R}^{d}\right)\right) \subset$ $\operatorname{Lip}\left(\mathbb{R}^{d}\right)$, for any $1 \leq k \leq n$. In this case, we have $\left(u_{k}\right)_{0 \leq k \leq n} \in \operatorname{Lip}\left(\mathbb{R}^{d}\right)^{n+1}$, and for any $0 \leq k \leq n$, we have the almost sure estimate

$$
\begin{aligned}
\left|u_{k}-\widehat{u}_{k}\right|\left(\widehat{X}_{k}\right) \leq & L\left(M_{k+1}\left(u_{k+1}\right)\right)\left|X_{k}-\widehat{X}_{k}\right| \\
& +\sum_{l=k+1}^{n-1}\left(L\left(u_{l}\right)+L\left(M_{l+1}\left(u_{l+1}\right)\right)\right) \mathbb{E}\left(\left|X_{l}-\widehat{X}_{l}\right|^{p} \mid \widehat{X}_{k}\right)^{\frac{1}{p}} \\
& +L\left(f_{n}\right)\left[\mathbb{E}\left(\left|X_{n}-\widehat{X}_{n}\right|^{p} \mid \widehat{X}_{k}\right)\right]^{\frac{1}{p}} .
\end{aligned}
$$

Proof:

Using the decomposition

$$
\widehat{u}_{k}\left(\widehat{X}_{k}\right)-u_{k}\left(X_{k}\right)=\left[\widehat{u}_{k}\left(\widehat{X}_{k}\right)-u_{k}\left(\widehat{X}_{k}\right)\right]+\left[u_{k}\left(\widehat{X}_{k}\right)-u_{k}\left(X_{k}\right)\right]
$$

we have that

$$
\left|u_{k}\left(\widehat{X}_{k}\right)-u_{k}\left(X_{k}\right)\right| \leq L\left(u_{k}\right)\left|\widehat{X}_{k}-X_{k}\right| .
$$

then the proof is ended by

$$
\begin{aligned}
\left|\widehat{u}_{k}\left(\widehat{\xi}_{k}\right)-u_{k}\left(X_{k}\right)\right| \leq & L\left(f_{n}\right)\left[\mathbb{E}\left(\left|X_{n}-\widehat{X}_{n}\right|^{p} \mid \widehat{X}_{k}\right)\right]^{\frac{1}{p}} \\
& +\sum_{l=k}^{n-1}\left(L\left(u_{l}\right)+L\left(M_{l+1}\left(u_{l+1}\right)\right)\right) \mathbb{E}\left(\left|X_{l}-\widehat{X}_{l}\right|^{p} \mid \widehat{X}_{k}\right)^{\frac{1}{p}}
\end{aligned}
$$




\section{Monte Carlo importance sampling approxima- tion schemes}

\subsection{Path space models}

The choice of non homogeneous state spaces $E_{n}$ is not innocent. In several application areas the underlying Markov model is a path-space Markov chain

$$
X_{n}=\left(X_{0}^{\prime}, \ldots, X_{n}^{\prime}\right) \in E_{n}=\left(E_{0}^{\prime} \times \ldots \times E_{n}^{\prime}\right) .
$$

The elementary prime variables $X_{n}^{\prime}$ represent an elementary Markov chain with Markov transitions $M_{k}^{\prime}\left(x_{k-1}, d x_{k}^{\prime}\right)$ from $E_{k-1}^{\prime}$ into $E_{k}^{\prime}$. In this situation, the historical process $X_{n}$ can be seen as a Markov chain with transitions given for any $x_{k-1}=\left(x_{0}^{\prime}, \ldots, x_{k-1}^{\prime}\right) \in E_{k-1}$ and $y_{k}=\left(y_{0}^{\prime}, \ldots, y_{k}^{\prime}\right) \in E_{k}$ by the following formula

$$
M_{k}\left(x_{k-1}, d y_{k}\right)=\delta_{x_{k-1}}\left(d y_{k-1}\right) M_{k}^{\prime}\left(y_{k-1}^{\prime}, d y_{k}^{\prime}\right) .
$$

This path space framework is, for instance, well suited when dealing with path dependent options as Asian options.

Besides, this path space framework is also well suited for the analysis of Snell envelopes under different probability measures. To fix the ideas, we associate with the latter a canonical Markov chain $\left(\Omega, \mathcal{F},\left(X_{n}^{\prime}\right)_{n \geq 0}, \mathbb{P}_{\eta_{0}^{\prime}}^{\prime}\right)$ with initial distribution $\eta_{0}^{\prime}$ on $E_{0}^{\prime}$, and Markov transitions $M_{n}^{\prime}$ from $E_{n-1}^{\prime}$ into $E_{n}^{\prime}$. We use the notation $\mathbb{E}_{\mathbb{P}_{\eta_{0}^{\prime}}^{\prime}}$ to denote the expectations with respect to $\mathbb{P}_{\eta_{0}^{\prime}}^{\prime}$. We further assume that there exists a sequence of measures $\left(\eta_{k}\right)_{0 \leq k \leq n}$ on the state spaces $\left(E_{k}^{\prime}\right)_{0 \leq k \leq n}$ such that

$$
\eta_{0}^{\prime} \sim \eta_{0} \quad \text { and } \quad M_{k}^{\prime}\left(x_{k-1}^{\prime}, .\right) \sim \eta_{k}
$$

for any $x_{k-1}^{\prime} \in E_{k-1}^{\prime}$, and $1 \leq k \leq n$. We let $\left(\Omega, \mathcal{F},\left(X_{n}^{\prime}\right)_{n \geq 0}, \mathbb{P}_{\eta_{0}}\right)$ be the canonical space associated with a sequence of independent random variables $X_{k}^{\prime}$ with distribution $\eta_{k}$ on the state space $E_{k}^{\prime}$, with $k \geq 1$. Under the probability measure $\mathbb{P}_{\eta_{0}}$, the historical process $X_{n}=\left(X_{0}^{\prime}, \ldots, X_{n}^{\prime}\right)$ can be seen as a Markov chain with transitions

$$
M_{k}\left(x_{k-1}, d y_{k}\right)=\delta_{x_{k-1}}\left(d y_{k-1}\right) \eta_{k}\left(d y_{k}^{\prime}\right) .
$$

By construction, for any integrable function $f_{k}^{\prime}$ on $E_{k}^{\prime}$, we have

$$
\mathbb{E}_{\mathbb{P}_{\eta_{0}^{\prime}}^{\prime}}\left(f_{n}^{\prime}\left(X_{n}^{\prime}\right)\right)=\mathbb{E}_{\mathbb{P}_{\eta_{0}}}\left(f_{n}\left(X_{n}\right)\right)
$$

with the collection of functions $f_{k}$ on $E_{k}$ given for any $x_{k}=\left(x_{0}^{\prime}, \ldots, x_{k}^{\prime}\right) \in E_{k}$ by

$f_{k}\left(x_{k}\right)=f_{k}^{\prime}\left(x_{k}^{\prime}\right) \times \frac{d \mathbb{P}_{k}^{\prime}}{d \mathbb{P}_{k}}\left(x_{k}\right) \quad$ with $\quad \frac{d \mathbb{P}_{k}^{\prime}}{d \mathbb{P}_{k}}\left(x_{k}\right)=\frac{d \eta_{0}^{\prime}}{d \eta_{0}}\left(x_{0}^{\prime}\right) \prod_{1 \leq l \leq k} \frac{d M_{l}^{\prime}\left(x_{l-1}^{\prime}, .\right)}{d \eta_{l}}\left(x_{l}^{\prime}\right)$.

Proposition 4.1 The Snell envelopes $u_{k}$ and $u_{k}^{\prime}$ associated with the pairs $\left(f_{k}^{\prime}, M_{k}^{\prime}\right)$ and $\left(f_{k}, M_{k}\right)$ are given for any $0 \leq k<n$ by the backward recursions

$u_{k}^{\prime}=f_{k}^{\prime} \vee M_{k+1}^{\prime}\left(u_{k+1}^{\prime}\right) \quad$ and $u_{k}=f_{k} \vee M_{k+1}\left(u_{k+1}\right) \quad$ with $\quad\left(u_{n}^{\prime}, u_{n}\right)=\left(f_{n}^{\prime}, f_{n}\right)$. 
These functions are connected by the following formula

$$
\forall 0 \leq k \leq n \quad \forall x_{k}=\left(x_{0}^{\prime}, \ldots, x_{k}^{\prime}\right) \in E_{k} \quad u_{k}\left(x_{k}\right)=u_{k}^{\prime}\left(x_{k}^{\prime}\right) \times \frac{d \mathbb{P}_{k}^{\prime}}{d \mathbb{P}_{k}}\left(x_{k}\right) .
$$

\section{Proof:}

The first assertion is a simple consequence of the definition of a Snell envelope, and formula (4.4) is easily derived using the fact that

$$
u_{k}^{\prime}\left(x_{k}^{\prime}\right)=f_{k}^{\prime}\left(x_{k}^{\prime}\right) \vee\left(\int_{E_{k+1}^{\prime}} \eta_{k+1}\left(d x_{k+1}^{\prime}\right) \frac{d M_{k+1}^{\prime}\left(x_{k}^{\prime}, .\right)}{d \eta_{k+1}}\left(x_{k+1}^{\prime}\right) u_{k+1}^{\prime}\left(x_{k+1}^{\prime}\right)\right) \text {. }
$$

This ends the proof of the proposition.

Under condition (4.2), the above proposition shows that the calculation of the Snell envelope associated with a given pair of functions and Markov transitions $\left(f_{k}^{\prime}, M_{k}^{\prime}\right)$ reduces to that of the path space models associated with sequence of independent random variables with distributions $\eta_{n}$. More formally, the restriction $\mathbb{P}_{\eta_{0}, n}$ of reference measure $\mathbb{P}_{\eta_{0}}$ to the $\sigma$-field $\mathcal{F}_{n}$ generated by the canonical random sequence $\left(X_{k}^{\prime}\right)_{0<k<n}$ is given by the the tensor product measure $\mathbb{P}_{\eta_{0}, n}=\otimes_{k=0}^{n} \eta_{k}$. Nevertheless, under these reference distributions the numerical solving of the backward recursion stated in the above proposition still involves integrations w.r.t. the measures $\eta_{k}$. These equations can be solved if we replace these measures by some sequence of (possibly random) measures $\widehat{\eta}_{k}$ with finite support on some reduced measurable subset $\widehat{E}_{k}^{\prime} \subset E_{k}^{\prime}$, with a reasonably large and finite cardinality. We extend $\widehat{\eta}_{k}$ to the whole space $E_{k}^{\prime}$ by setting $\widehat{\eta}_{k}\left(E_{k}^{\prime}-\widehat{E}_{k}^{\prime}\right)=0$.

We let $\widehat{\mathbb{P}}_{\widehat{\eta}_{0}^{\prime}}$ be the distribution of a sequence of independent random variables $\widehat{\xi}_{k}^{\prime}$ with distribution $\widehat{\eta}_{k}$ on the state space $\widehat{E}_{k}^{\prime}$, with $k \geq 1$. Under the probability measure $\widehat{\mathbb{P}}_{\widehat{\eta}_{0}^{\prime}}$, the historical process $X_{n}=\left(X_{0}^{\prime}, \ldots, X_{n}^{\prime}\right)$ can now be seen as a Markov chain taking values in the path spaces

$$
\widehat{E}_{k}:=\left(\widehat{E}_{0}^{\prime} \times \ldots \times \widehat{E}_{k}^{\prime}\right),
$$

with Markov transitions given for any $x_{k-1}=\left(x_{0}^{\prime}, \ldots, x_{k-1}^{\prime}\right) \in \widehat{E}_{k-1}$ and $y_{k}=$ $\left(y_{0}^{\prime}, \ldots, y_{k}^{\prime}\right) \in \widehat{E}_{k}$ by the following formula

$$
\widehat{M}_{k}\left(x_{k-1}, d y_{k}\right)=\delta_{x_{k-1}}\left(d y_{k-1}\right) \widehat{\eta}_{k}\left(d y_{k}^{\prime}\right) .
$$

Notice that the restriction $\widehat{\mathbb{P}}_{\widehat{\eta}_{0}^{\prime}, n}$ of these approximated reference measure $\widehat{\mathbb{P}}_{\widehat{\eta}_{0}^{\prime}}$ to the $\sigma$-field $\mathcal{F}_{n}$ generated by the canonical random sequence $\left(X_{k}^{\prime}\right)_{0 \leq k \leq n}$ is now given by the the tensor product measure $\widehat{\mathbb{P}}_{\widehat{\eta}_{0}^{\prime}, n}=\otimes_{k=0}^{n} \widehat{\eta}_{k}$.

We let $\widehat{u}_{k}$ be the Snell envelope on the path space $\widehat{E}_{k}$, associated with the pair $\left(\widehat{f}_{k}, \widehat{M}_{k}\right)$, with the sequence of functions $\widehat{f}_{k}=f_{k}$ given in (4.3). By construction, for any $0 \leq k \leq n$, and any path $x_{k}=\left(x_{0}^{\prime}, \ldots, x_{k}^{\prime}\right) \in \widehat{E}_{k}$, we have

$$
\widehat{u}_{k}\left(x_{k}\right)=\widehat{u}_{k}^{\prime}\left(x_{k}^{\prime}\right) \times \frac{d \mathbb{P}_{k}^{\prime}}{d \mathbb{P}_{k}}\left(x_{k}\right)
$$


with the collection of functions $\left(\widehat{u}_{k}^{\prime}\right)_{0 \leq k \leq n}$ on the state spaces $\left(E_{k}^{\prime}\right)_{0 \leq k \leq n}$ given by the backward recursions

$$
\widehat{u}_{k}^{\prime}\left(x_{k}^{\prime}\right)=f_{k}^{\prime}\left(x_{k}^{\prime}\right) \vee\left(\int_{\widehat{E}_{k+1}^{\prime}} \widehat{M}_{k+1}^{\prime}\left(x_{k}^{\prime}, d x_{k+1}^{\prime}\right) \widehat{u}_{k+1}^{\prime}\left(x_{k+1}^{\prime}\right)\right),
$$

with the random integral operator $\widehat{M}_{k}^{\prime}$ from $E_{k}^{\prime}$ into $\widehat{E}_{k+1}^{\prime}$ defined below

$$
\widehat{M}_{k+1}^{\prime}\left(x_{k}^{\prime}, d x_{k+1}^{\prime}\right)=\widehat{\eta}_{k+1}\left(d x_{k+1}^{\prime}\right) R_{k+1}\left(x_{k}^{\prime}, x_{k+1}^{\prime}\right)
$$

with the Radon Nikodym derivatives $R_{k+1}\left(x_{k}^{\prime}, x_{k+1}^{\prime}\right)=\frac{d M_{k+1}^{\prime}\left(x_{k}^{\prime}, \cdot\right)}{d \eta_{k+1}}\left(x_{k+1}^{\prime}\right)$.

\subsection{Broadie-Glasserman models}

We consider the path space models associated to the changes of measures presented in Sub-section 4.1. We use the same notation as in there. We further assume that $\widehat{\eta}_{k}=\frac{1}{N} \sum_{i=1}^{N} \delta_{\xi_{k}^{i}}$ is the occupation measure associated with a sequence of independent random variables $\xi_{k}:=\left(\xi_{k}^{i}\right)_{1 \leq i \leq N}$ with common distribution $\eta_{k}$ on $\widehat{E}_{k}^{\prime}=E_{k}^{\prime}$. We further assume that $\left(\xi_{k}\right)_{0 \leq k \leq n}$ are independent. This Monte Carlo type model has been introduced in 1997 by M. Broadie, and P. Glasserman (see for instance [5], and references therein). We let $\widehat{\mathbb{E}}$ be the expectation operator associated with this additional level of randomness, and we set $\widehat{\mathbb{E}}_{\mathbb{P}_{\eta_{0}}}:=\widehat{\mathbb{E}} \otimes \mathbb{E}_{\mathbb{P}_{\eta_{0}}}$.

In this situation, we observe that

$$
\left(M_{k+1}^{\prime}-\widehat{M}_{k+1}^{\prime}\right)\left(x_{k}^{\prime}, d x_{k+1}^{\prime}\right)=\frac{1}{\sqrt{N}} \widehat{V}_{k+1}\left(d x_{k+1}^{\prime}\right) R_{k+1}\left(x_{k}^{\prime}, x_{k+1}^{\prime}\right),
$$

with the random fields $\widehat{V}_{k+1}:=\sqrt{N}\left[\eta_{k+1}-\widehat{\eta}_{k+1}\right]$. From these observations, we readily prove that the approximation operators $\widehat{M}_{k+1}^{\prime}$ are unbiased, in the sense that

$$
\forall 0 \leq k \leq l \quad \forall x_{l}^{\prime} \in E_{l} \quad \widehat{\mathbb{E}}_{\mathbb{P}_{\eta_{0}}}\left(\widehat{M}_{k, l}^{\prime}(f)\left(x_{l}^{\prime}\right) \mid \mathcal{F}_{k}\right)=M_{k, l}^{\prime}(f)\left(x_{l}^{\prime}\right),
$$

for any bounded function $f$ on $E_{l+1}$. Furthermore, for any even integer $p \geq 1$, we have

$$
\sqrt{N} \widehat{\mathbb{E}}_{\mathbb{P}_{\eta_{0}}}\left(\left|\left[M_{l+1}^{\prime}-\widehat{M}_{l+1}^{\prime}\right](f)\left(x_{l}^{\prime}\right)\right|^{p}\right)^{\frac{1}{p}} \leq 2 a(p) \eta_{l+1}\left[\left(R_{l+1}\left(x_{l}^{\prime}, .\right) f\right)^{p}\right]^{\frac{1}{p}} .
$$

The above estimate is valid as soon as the r.h.s. in the above inequality is well defined.

We are now in position to state and prove the following theorem.

Theorem 4.2 For any integer $p \geq 1$, we denote by $p^{\prime}$ the smallest even integer greater than $p$. Then for any time horizon $0 \leq k \leq n$, and any $x_{k}^{\prime} \in E_{k}^{\prime}$, we have

$$
\begin{aligned}
& \sqrt{N} \widehat{\mathbb{E}}_{\mathbb{P}_{\eta_{0}}}\left(\left|u_{k}^{\prime}\left(x_{k}^{\prime}\right)-\widehat{u}_{k}^{\prime}\left(x_{k}^{\prime}\right)\right|^{p}\right)^{\frac{1}{p}}(4.7) \\
& \leq 2 a(p) \sum_{k \leq l<n}\left\{\int M_{k, l}^{\prime}\left(x_{k}^{\prime}, d x_{l}^{\prime}\right) \eta_{l+1}\left[\left(R_{l+1}\left(x_{l}^{\prime}, .\right) u_{l+1}^{\prime}\right)^{p^{\prime}}\right]\right\}^{\frac{1}{p^{\prime}}} .
\end{aligned}
$$


Notice that, as stated in the introduction, this result implies exponential rate of convergence in probability. Hence, this allows to improve noticeably existing convergence results stated in [5], with no rate of convergence, and in [1] with a polynomial rate of convergence in probability.

Proof:

For any even integers $p \geq 1$, any $0 \leq k \leq l$, any measurable function $f$ on $E_{l+1}$, and any $x_{k} \in E_{k}^{\prime}$, using the generalized Minkowski inequality we find that

$$
\begin{aligned}
& \sqrt{N} \widehat{\mathbb{E}}_{\mathbb{P}_{\eta_{0}}}\left(\left|\widehat{M}_{k, l}^{\prime}\right|\left[M_{l+1}^{\prime}-\widehat{M}_{l+1}^{\prime}\right](f)\left|\left(x_{k}^{\prime}\right)\right|^{p} \mid \mathcal{F}_{l}\right)^{\frac{1}{p}} \\
& \quad \leq 2 a(p) \int \widehat{M}_{k, l}^{\prime}\left(x_{k}^{\prime}, d x_{l}^{\prime}\right) \eta_{l+1}\left[\left(R_{l+1}\left(x_{l}^{\prime}, .\right) f\right)^{p}\right]^{\frac{1}{p}} .
\end{aligned}
$$

By the unbias property (4.6), we conclude that

$$
\begin{aligned}
\sqrt{N} \widehat{\mathbb{E}}_{\mathbb{P}_{\eta_{0}}}\left(\left|\widehat{M}_{k, l}^{\prime}\right|\left[M_{l+1}^{\prime}-\widehat{M}_{l+1}^{\prime}\right](f)\left|\left(x_{k}^{\prime}\right)\right|^{p}\right)^{\frac{1}{p}} \\
\leq 2 a(p)\left\{\int M_{k, l}^{\prime}\left(x_{k}^{\prime}, d x_{l}^{\prime}\right) \eta_{l+1}\left[\left(R_{l+1}\left(x_{l}^{\prime}, .\right) f\right)^{p}\right]\right\}^{1 / p} .
\end{aligned}
$$

For odd integers $p=2 q+1$, with $q \geq 0$, we use the fact that

$$
\mathbb{E}\left(Y^{2 q+1}\right)^{2} \leq \mathbb{E}\left(Y^{2 q}\right) \mathbb{E}\left(Y^{2(q+1)}\right) \quad \text { and } \quad \mathbb{E}\left(Y^{2 q}\right) \leq \mathbb{E}\left(Y^{2(q+1)}\right)^{\frac{q}{q+1}},
$$

for any nonnegative random variable $Y$ and

$$
(2(q+1))_{q+1}=2(2 q+1)_{q+1} \quad \text { and } \quad(2 q)_{q}=(2 q+1)_{q+1} /(2 q+1),
$$

so that

$$
\begin{gathered}
a(2 q)^{2 q} a(2(q+1))^{2(q+1)} \leq 2^{-(2 q+1)}(2 q+1)_{q+1}^{2} /(q+1 / 2)=\left(a(2 q+1)^{2 q+1}\right)^{2} \\
N \widehat{\mathbb{E}}_{\mathbb{P}_{\eta_{0}}}\left(\left|\widehat{M}_{k, l}^{\prime}\right|\left[M_{l+1}^{\prime}-\widehat{M}_{l+1}^{\prime}\right](f)\left|\left(x_{k}^{\prime}\right)\right|^{2 q+1}\right)^{2} \\
\leq\left(2^{(2 q+1)} a(2 q+1)^{2 q+1}\right)^{2} \int M_{k, l}^{\prime}\left(x_{k}^{\prime}, d x_{l}^{\prime}\right) \eta_{l+1}\left[\left(R_{l+1}\left(x_{l}^{\prime}, .\right) f\right)^{2(q+1)}\right]^{\frac{q}{q+1}} \cdot \\
\times \int M_{k, l}^{\prime}\left(x_{k}^{\prime}, d x_{l}^{\prime}\right) \eta_{l+1}\left[\left(R_{l+1}\left(x_{l}^{\prime}, .\right) f\right)^{2(q+1)}\right]
\end{gathered}
$$

Using the fact that $\mathbb{E}\left(Y^{\frac{q}{q+1}}\right) \leq \mathbb{E}(Y)^{\frac{q}{q+1}}$, we prove that the r.h.s. term in the above display is upper bounded by

$$
\left(2^{(2 q+1)} a(2 q+1)^{2 q+1}\right)^{2}\left\{\int M_{k, l}^{\prime}\left(x_{k}^{\prime}, d x_{l}^{\prime}\right) \eta_{l+1}\left[\left(R_{l+1}\left(x_{l}^{\prime}, .\right) f\right)^{2(q+1)}\right]\right\}^{2\left(1-\frac{1}{2(q+1)}\right)},
$$

from which we conclude that

$$
\begin{aligned}
& \sqrt{N} \widehat{\mathbb{E}}_{\mathbb{P}_{\eta_{0}}}\left(\left|\widehat{M}_{k, l}^{\prime}\right|\left[M_{l+1}^{\prime}-\widehat{M}_{l+1}^{\prime}\right](f)\left|\left(x_{k}^{\prime}\right)\right|^{2 q+1}\right)^{\frac{1}{2 q+1}} \\
& \leq 2 a(2 q+1)\left\{\int M_{k, l}^{\prime}\left(x_{k}^{\prime}, d x_{l}^{\prime}\right) \eta_{l+1}\left[\left(R_{l+1}\left(x_{l}^{\prime}, \cdot\right) f\right)^{2(q+1)}\right]\right\}^{\frac{1}{2(q+1)}} .
\end{aligned}
$$

$\mathrm{RR} \mathrm{n}^{\circ} 7303$ 
This ends the proof of the theorem.

The $\mathbb{L}_{p}$-mean error estimates stated in Theorem 4.2 are expressed in terms of $\mathbb{L}_{p^{\prime}}$ norms of Snell envelope functions and Radon Nikodym derivatives. The terms in the r.h.s. of (4.7) have the following interpretation:

$$
\begin{aligned}
\int M_{k, l}^{\prime}\left(x_{k}^{\prime}, d x_{l}^{\prime}\right) \eta_{l+1}\left[\left(R_{l+1}\left(x_{l}^{\prime}, .\right) u_{l+1}\right)^{p^{\prime}}\right] \\
=\mathbb{E}\left[\left(R_{l+1}\left(X_{l}^{\prime}, \xi_{l+1}^{1}\right) u_{l+1}\left(\xi_{l+1}^{1}\right)\right)^{p^{\prime}} \mid X_{k}^{\prime}=x_{k}^{\prime}\right] .
\end{aligned}
$$

In the above display, $\mathbb{E}($.$) stands for the expectation w.r.t. some reference$ probability measure under which $X_{l}^{\prime}$ is a Markov chain with transitions $M_{l}^{\prime}$, and $\xi_{l+1}^{1}$ is an independent random variable with distribution $\eta_{l+1}$. Loosely speaking, the above quantities can be very large when the sampling distributions $\eta_{l+1}$ are far from the distribution of the random states $X_{l+1}^{\prime}$ of the reference Markov chain at time $(l+1)$. Next we provide an original strategy that allows for instance to take $\eta_{l+1}=\operatorname{Law}\left(X_{l+1}^{\prime}\right)$ as the sampling distribution, even if $R_{l+1}$ is not known (i.e. cannot be evaluated at any point of $E_{l+1}$ ). In the sequel, we consider $N$ independent copies $\left(\xi_{0}^{i}, \cdots \xi_{n}^{i}\right)_{1<i<N}$ of the Markov chain $\left(X_{0}^{\prime}, X_{1}^{\prime}, \cdots X_{n}^{\prime}\right)$, from the origin $k=0$ up to the final time horizon $k=n$. Then, for all $k=0, \cdots n$, we define the associated occupation measure $\widehat{\eta}_{k}=\frac{1}{N} \sum_{i=1}^{N} \delta_{\xi_{k}^{i}}$. For all $k=0, \cdots n$, we let $\mathcal{F}_{k}$ be the sigma field generated by the random sequence $\left(\xi_{l}\right)_{0 \leq l \leq k}$.

We also assume that the Markov transitions $M_{n}^{\prime}\left(x_{n-1}^{\prime}, d x_{n}^{\prime}\right)$ are absolutely continuous with respect to some measures $\lambda_{n}\left(d x_{n}^{\prime}\right)$ on $E_{n}^{\prime}$ and we have

$(H)_{0} \quad \forall\left(x_{n-1}^{\prime}, x_{n}^{\prime}\right) \in\left(E_{n-1}^{\prime} \times E_{n}^{\prime}\right) \quad H_{n}\left(x_{n-1}^{\prime}, x_{n}^{\prime}\right)=\frac{d M_{n}^{\prime}\left(x_{n-1}^{\prime}, .\right)}{d \lambda_{n}}\left(x_{n}^{\prime}\right)>0$,

where $H_{n}$ is supposed to be known up to a normalizing constant. In this situation, we have $\eta_{k+1} \ll \lambda_{k+1}$, with the Radon Nikodym derivative given below

$$
\eta_{k+1}\left(d x_{k+1}^{\prime}\right)=\eta_{k} M_{k+1}^{\prime}\left(d x_{k+1}^{\prime}\right)=\eta_{k}\left(H_{k+1}\left(., x_{k+1}^{\prime}\right)\right) \lambda_{k+1}\left(d x_{k+1}^{\prime}\right) .
$$

Also notice that the backward recursion of the Snell envelope $u_{k}^{\prime}$ can be rewritten as follows

$$
\begin{aligned}
u_{k}^{\prime}\left(x_{k}^{\prime}\right) & =f_{k}^{\prime}\left(x_{k}^{\prime}\right) \vee\left(\int_{E_{k+1}^{\prime}} \eta_{k+1}\left(d x_{k+1}^{\prime}\right) \frac{d M_{k+1}^{\prime}\left(x_{k}^{\prime}, .\right)}{d \eta_{k+1}}\left(x_{k+1}^{\prime}\right) u_{k+1}^{\prime}\left(x_{k+1}^{\prime}\right)\right) \\
& =f_{k}^{\prime}\left(x_{k}^{\prime}\right) \vee\left(\int_{E_{k+1}^{\prime}} \eta_{k+1}\left(d x_{k+1}^{\prime}\right) \frac{H_{k+1}\left(x_{k}^{\prime}, x_{k+1}^{\prime}\right)}{\eta_{k}\left(H_{k+1}\left(., x_{k+1}^{\prime}\right)\right)} u_{k+1}^{\prime}\left(x_{k+1}^{\prime}\right)\right) .
\end{aligned}
$$

Arguing as in (4.5), we define the approximated Snell envelope $\left(\widehat{u}_{k}^{\prime}\right)_{0 \leq k \leq n}$ on the state spaces $\left(E_{k}^{\prime}\right)_{0 \leq k \leq n}$ by setting

$$
\widehat{u}_{k}^{\prime}\left(x_{k}^{\prime}\right)=f_{k}^{\prime}\left(x_{k}^{\prime}\right) \vee\left(\int_{\widehat{E}_{k+1}^{\prime}} \widehat{M}_{k+1}^{\prime}\left(x_{k}^{\prime}, d x_{k+1}^{\prime}\right) \widehat{u}_{k+1}^{\prime}\left(x_{k+1}^{\prime}\right)\right),
$$

with the random integral operator $\widehat{M}^{\prime}$ from $E_{k}$ into $\widehat{E}_{k+1}$ defined below

$$
\begin{aligned}
\widehat{M}_{k+1}^{\prime}\left(x_{k}^{\prime}, d x_{k+1}^{\prime}\right) & =\widehat{\eta}_{k+1}\left(d x_{k+1}^{\prime}\right) \frac{d M_{k+1}^{\prime}\left(x_{k}^{\prime}, .\right)}{d \widehat{\eta}_{k} M_{k+1}^{\prime}}\left(x_{k+1}^{\prime}\right) \\
& =\widehat{\eta}_{k+1}\left(d x_{k+1}^{\prime}\right) \frac{H_{k+1}\left(x_{k}^{\prime}, x_{k+1}^{\prime}\right)}{\widehat{\eta}_{k}\left(H_{k+1}\left(., x_{k+1}^{\prime}\right)\right)}
\end{aligned}
$$


By construction, these random approximation operators $\widehat{M}_{k+1}^{\prime}$ satisfy the unbias property stated in (4.6), and we have

$$
\left(M_{k+1}^{\prime}-\widehat{M}_{k+1}^{\prime}\right)\left(x_{k}^{\prime}, d x_{k+1}^{\prime}\right)=\frac{1}{\sqrt{N}} \widehat{V}_{k+1}\left(d x_{k+1}^{\prime}\right) \widehat{R}_{k+1}\left(x_{k}^{\prime}, x_{k+1}^{\prime}\right),
$$

with the random fields $\widehat{V}_{k+1}$ and the $\mathcal{F}_{k}$-measurable random functions $\widehat{R}_{k+1}$ defined below

$$
\widehat{V}_{k+1}:=\sqrt{N}\left[\widehat{\eta}_{k} M_{k+1}^{\prime}-\widehat{\eta}_{k+1}\right] \quad \text { and } \quad \widehat{R}_{k+1}\left(x_{k}^{\prime}, x_{k+1}^{\prime}\right):=\frac{H_{k+1}\left(x_{k}^{\prime}, x_{k+1}^{\prime}\right)}{\widehat{\eta}_{k}\left(H_{k+1}\left(., x_{k+1}^{\prime}\right)\right)} .
$$

Furthermore, for any even integer $p \geq 1$, and any measurable function $f$ on $E_{l}$ we have

$\sqrt{N} \widehat{\mathbb{E}}_{\mathbb{P}_{\eta_{0}}}\left(\left|\left[M_{l+1}^{\prime}-\widehat{M}_{l+1}^{\prime}\right](f)\left(x_{l}^{\prime}\right)\right|^{p} \mid \mathcal{F}_{l}\right)^{\frac{1}{p}} \leq 2 a(p) \widehat{\eta}_{l} M_{l+1}^{\prime}\left[\left(\widehat{R}_{l+1}\left(x_{l}^{\prime}, .\right) f\right)^{p}\right]^{\frac{1}{p}}$.

The above estimate is valid as soon as the r.h.s. in the above inequality is well defined. For instance, assuming that

$$
\left\|M_{l+1}^{\prime}\left(u_{l+1}^{2 p}\right)\right\|<\infty
$$

and

$$
\sup _{x_{l}^{\prime}, y_{l}^{\prime} \in E_{l}^{\prime}} \frac{H_{l+1}\left(x_{l}^{\prime}, x_{l+1}^{\prime}\right)}{H_{l+1}\left(y_{l}^{\prime}, x_{l+1}^{\prime}\right)} \leq h_{l+1}\left(x_{l+1}^{\prime}\right) \text { with }\left\|M_{l+1}^{\prime}\left(h_{l+1}^{2 p}\right)\right\|<\infty
$$

we find that

$$
\begin{aligned}
& \sqrt{N} \mathbb{E}\left(\left|\left[M_{l+1}^{\prime}-\widehat{M}_{l+1}^{\prime}\right]\left(u_{l+1}^{\prime}\right)\left(x_{l}^{\prime}\right)\right|^{p} \mid \mathcal{F}_{l}\right)^{\frac{1}{p}} \\
& \quad \leq 2 a(p)\left(\left\|M_{l+1}^{\prime}\left(h_{l+1}^{2 p}\right)\right\|\left\|M_{l+1}^{\prime}\left(\left(u_{l+1}^{\prime}\right)^{2 p}\right)\right\|\right)^{\frac{1}{2 p}} .
\end{aligned}
$$

Rephrasing the proof of Theorem 4.2, we prove the following result.

Theorem 4.3 Under the conditions $(H)_{0}$ and $(H)_{1}$ stated above, for any even integer $p>1$, any $0 \leq k \leq n$, and $x_{k}^{\prime} \in E_{k}^{\prime}$, we have

$$
\begin{aligned}
& \sqrt{N} \mathbb{E}\left(\left|u_{k}^{\prime}\left(x_{k}^{\prime}\right)-\widehat{u}_{k}^{\prime}\left(x_{k}^{\prime}\right)\right|^{p}\right)^{\frac{1}{p}} \\
& \leq 2 a(p) \sum_{k \leq l<n}\left(\left\|M_{l+1}^{\prime}\left(h_{l+1}^{2 p}\right)\right\|\left\|M_{l+1}^{\prime}\left(\left(u_{l+1}^{\prime}\right)^{2 p}\right)\right\|\right)^{\frac{1}{2 p}}
\end{aligned}
$$

In the end of this subsection, recovering and extending results from [5], it is interesting to point out that both the Broadie-Glasserman estimator and this new BG type adapted estimator have positive bias.

Proposition 4.4 For any $0 \leq k \leq n$ and any $x_{k}^{\prime} \in E_{k}^{\prime}$

$$
\mathbb{E}\left(\widehat{u}_{k}^{\prime}\left(x_{k}^{\prime}\right)\right) \geq u_{k}^{\prime}\left(x_{k}^{\prime}\right) \text {. }
$$

\section{Proof:}

This inequality can be proved easily by a simple backward induction. The terminal condition $\widehat{u}_{n}^{\prime}=u_{n}^{\prime}$ implies directly the inequality on instant $n$. Assuming the inequality holds true in instant $k$, then Jensen's inequality implies that

$$
\begin{aligned}
\mathbb{E}\left(\widehat{u}_{k}^{\prime}\left(x_{k}^{\prime}\right)\right) & \geq f_{k}\left(x_{k}^{\prime}\right) \vee \mathbb{E}\left(\widehat{M}_{k+1}^{\prime}\left(\widehat{u}_{k+1}^{\prime}\right)\left(x_{k}^{\prime}\right)\right) \\
& \geq f_{k}\left(x_{k}^{\prime}\right) \vee M_{k+1} u_{k+1}^{\prime}\left(x_{k}^{\prime}\right)=u_{k}^{\prime}\left(x_{k}^{\prime}\right) .
\end{aligned}
$$


This ends the proof of the proposition.

\section{A genealogical tree based model}

\subsection{Neutral genetic models}

Using the notation of Sub-section 4.1. we set

$$
X_{n}=\left(X_{0}^{\prime}, \ldots, X_{n}^{\prime}\right) \in E_{n}=\left(E_{0}^{\prime} \times \ldots \times E_{n}^{\prime}\right),
$$

We further assume that the state spaces $E_{n}^{\prime}$ are finite. We denote by $\eta_{k}$ the distribution of the path-valued random variable $X_{k}$ on $E_{k}$, with $0 \leq k \leq n$.

We also set $M_{k}^{\prime}$ the Markov transition from $X_{k-1}^{\prime}$ to $X_{k}^{\prime}$, and $M_{k}$ the Markov transition from $X_{k-1}$ to $X_{k}$. In Sub-section 4.1, we have seen that

$M_{k}\left(\left(x_{0}^{\prime}, \ldots, x_{k-1}^{\prime}\right), d\left(y_{0}^{\prime}, \ldots, y_{k}^{\prime}\right)\right)=\delta_{\left(x_{0}^{\prime}, \ldots, x_{k-1}^{\prime}\right)}\left(d\left(y_{0}^{\prime}, \ldots, y_{k-1}^{\prime}\right)\right) M_{k}^{\prime}\left(y_{k-1}^{\prime}, d y_{k}^{\prime}\right)$.

In the further development, we fix the final time horizon $n$, and for any $0 \leq k \leq$ $n$, we denote by $\pi_{k}$ the $k$-th coordinate mapping

$$
\pi_{k}: x_{n}=\left(x_{0}^{\prime}, \ldots, x_{n}^{\prime}\right) \in E_{n}=\left(E_{0}^{\prime} \times \ldots \times E_{n}^{\prime}\right) \mapsto \pi_{k}\left(x_{n}\right)=x_{k}^{\prime} \in E_{k}^{\prime} .
$$

In this notation, for any $0 \leq k<n, x_{k}^{\prime} \in E_{k}^{\prime}$ and any function $f \in \mathcal{B}\left(E_{k+1}^{\prime}\right)$, we have

$$
\eta_{n}=\operatorname{Law}\left(X_{0}^{\prime}, \ldots, X_{n}^{\prime}\right) \quad \text { and } \quad M_{k+1}^{\prime}(f)(x):=\frac{\eta_{n}\left(\left(1_{x} \circ \pi_{k}\right)\left(f \circ \pi_{k+1}\right)\right)}{\eta_{n}\left(\left(1_{x} \circ \pi_{k}\right)\right)}
$$

By construction, it is also readily checked that the flow of measure $\left(\eta_{k}\right)_{0 \leq k \leq n}$ also satisfies the following equation

$$
\forall 1 \leq k \leq n \quad \eta_{k}:=\Phi_{k}\left(\eta_{k-1}\right)
$$

with the linear mapping $\Phi_{k}\left(\eta_{k-1}\right):=\eta_{k-1} M_{k}$.

The genealogical tree based particle approximation associated with these recursion is defined in terms of a Markov chain $\xi_{k}^{(N)}=\left(\xi_{k}^{(i, N)}\right)_{1 \leq i \leq N_{k}}$ in the product state spaces $E_{k}^{N_{k}}$, where $N=\left(N_{k}\right)_{0 \leq k \leq N}$ is a given collection of integers.

$$
\mathbb{P}\left(\xi_{k}^{(N)}=\left(x_{k}^{1}, \ldots, x_{k}^{N_{k}}\right) \mid \xi_{k-1}\right)=\prod_{1 \leq i \leq N_{k}} \Phi_{k}\left(\frac{1}{N_{k-1}} \sum_{1 \leq i \leq N_{k-1}} \delta_{\xi_{k-1}^{i}}\right)\left(x_{k}^{i}\right)
$$

The initial particle system $\xi_{0}^{(N)}=\left(\xi_{0}^{(i, N)}\right)_{0 \leq i \leq N_{0}}$, is a sequence of $N_{0}$ i.i.d. random copies of $X_{0}$. We let $\mathcal{F}_{k}^{N}$ be the sigma-field generated by the particle approximation model from the origin, up to time $k$.

To simplify the presentation, when there is no confusion we suppress the population size parameter $N$, and we write $\xi_{k}$ and $\xi_{k}^{i}$ instead of $\xi_{k}^{(N)}$ and $\xi_{k}^{(i, N)}$. 
By construction, $\xi_{k}$ is a genetic type model with a neutral selection transition and a mutation type exploration

$$
\xi_{k} \in E_{k}^{N_{k}} \stackrel{\text { Selection }}{\longrightarrow} \widehat{\xi}_{k}:=\left(\widehat{\xi}_{k}^{i}\right)_{1 \leq i \leq \widehat{N}_{k}} \in E_{k}^{\widehat{N}_{k}} \stackrel{\text { Mutation }}{\longrightarrow} \xi_{k+1} \in E_{k+1}^{N_{k+1}}
$$

with $\widehat{N}_{k}:=N_{k+1}$.

During the selection transition, we select randomly $N_{k+1}$ path valued particles $\widehat{\xi}_{k}:=\left(\widehat{\xi}_{k}^{i}\right)_{1 \leq i<N_{k+1}}$ among the $N_{k}$ path valued particles $\xi_{k}=\left(\xi_{k}^{i}\right)_{1 \leq i \leq N_{k}}$. Sometimes, this elementary transition is called a neutral selection transition in the literature on genetic population models. During the mutation transition $\widehat{\xi}_{k} \rightsquigarrow \xi_{k}$, every selected path valued individual $\widehat{\xi}_{k}^{i}$ evolves randomly to a new path valued individual $\xi_{k+1}^{i}=x$ randomly chosen with the distribution $M_{k+1}\left(\widehat{\xi}_{k}^{i}, x\right)$, with $1 \leq i \leq \widehat{N}_{k}$. By construction, every particle is a path-valued random variable defined by

$$
\begin{aligned}
\xi_{k}^{i} & :=\left(\xi_{0, k}^{i}, \xi_{1, k}^{i}, \ldots, \xi_{k, k}^{i}\right) \\
\widehat{\xi}_{k}^{i} & :=\left(\widehat{\xi}_{0, k}^{i}, \widehat{\xi}_{1, k}^{i}, \ldots, \widehat{\xi}_{k, k}^{i}\right) \in E_{k}:=\left(E_{0}^{\prime} \times \ldots \times E_{k}^{\prime}\right) .
\end{aligned}
$$

By definition of the transition in path space, we also have that

$$
\begin{aligned}
& \xi_{k+1}^{i}=(\underbrace{\left(\xi_{0, k+1}^{i}, \xi_{1, k+1}^{i}, \ldots, \xi_{k, k+1}^{i}\right)}_{\|}, \xi_{k+1, k+1}^{i}) \\
& =(\overbrace{\left(\widehat{(\xi), ~}_{\hat{\xi}_{0, k}^{i},} \widehat{\xi}_{1, k}^{i}, \ldots, \quad \widehat{\xi}_{k, k}^{i}\right)}, \quad \xi_{k+1, k+1}^{i})=\left(\widehat{\xi}_{k}^{i}, \xi_{k+1, k+1}^{i}\right),
\end{aligned}
$$

where $\xi_{k+1, k+1}^{i}$ is a random variable with distribution $M_{k+1}^{\prime}\left(\widehat{\xi}_{k, k}^{i},.\right)$. In other words, the mutation transition $\widehat{\xi}_{k}^{i} \rightsquigarrow \xi_{k+1}^{i}$ simply consists in extending the selected path $\widehat{\xi}_{k}^{i}$ with an elementary move $\widehat{\xi}_{k, k}^{i} \rightsquigarrow \xi_{k+1, k+1}^{i}$ of the end point of the selected path.

From these observations, it is easy to check that the terminal random population model $\xi_{k, k}=\left(\xi_{k, k}^{i}\right)_{1 \leq i \leq N_{k}}$ and $\widehat{\xi}_{k, k}=\left(\widehat{\xi}_{k, k}^{i}\right)_{1 \leq i \leq N_{k+1}}$ is again defined as a genetic type Markov chain defined as above by replacing the pair $\left(E_{k}, M_{k}\right)$ by the pair $\left(E_{k}^{\prime}, M_{k}^{\prime}\right)$, with $1 \leq k \leq n$. The latter coincides with the mean field particle model associated with the time evolution of the $k$-th time marginals $\eta_{k}^{\prime}$ of the measures $\eta_{k}$ on $E_{k}^{\prime}$. Furthermore, the above path-valued genetic model coincide with the genealogical tree evolution model associated with the terminal state random variables.

We let $\eta_{k}^{N}$ and $\widehat{\eta}_{k}^{N}$ be the occupation measures of the genealogical tree model after the mutation and the selection steps; that is, we have that

$$
\eta_{k}^{N}:=\frac{1}{N_{k}} \sum_{1 \leq i \leq N_{k}} \delta_{\xi_{k}^{i}} \text { and } \quad \widehat{\eta}_{k}^{N}:=\frac{1}{\widehat{N}_{k}} \sum_{1 \leq i \leq \widehat{N}_{k}} \delta_{\widehat{\xi}_{k}^{i}} .
$$

In this notation, the selection transition $\xi_{k}, \rightsquigarrow \widehat{\xi}_{k}$ consists in choosing $\widehat{N}_{k}$ conditionally independent and identically distributed random paths $\widehat{\xi}_{k}^{i}$ with common 
distribution $\eta_{k}^{N}$. In other words, $\widehat{\eta}_{k}^{N}$ is the empirical measure associated with $\widehat{N}_{k}$ conditionally independent and identically distributed random paths $\widehat{\xi}_{k}^{i}$ with common distribution $\eta_{k}^{N}$. Also observe $\eta_{k}^{N}$ is the empirical measure associated with $N_{k}$ conditionally independent and identically distributed random paths $\xi_{k}^{i}$ with common distribution $\eta_{k-1}^{N} M_{k}$.

In practice, we can take $N_{0}=N_{1}=\ldots N_{n}=N$ when we do not have any information on the variance of $X_{k}$. In the case when we know the approximate variance of $X_{k}$, we can take a large $N_{k}$ when the variance of $X_{k}^{\prime}$ is large. To clarify the presentation, In the further development of the article we further assume that the particle model has a fixed population size $N_{k}=N$, for any $k \geq 0$.

In the sequel, the simulation of the path valued particle system $\left(\xi_{k}\right)_{0 \leq k \leq n}$ will be called the Forward step and is summarized in the following algorithm.

\subsubsection{Forward algorithm}

Initialization At time step $k=0$, generate $N$ i.i.d. random copies of $X_{0}$ and set $\xi_{0}=\left(\xi_{0}^{i}\right)_{0 \leq i \leq N}$.

At each time step $k=1, \cdots, n$

1. Selection: For each $i=1, \cdots, N$, generate independently an indice $I_{i} \in\{1, \cdots, N\}$ with probability $\mathcal{P}\left(I_{i}=j\right)=1 / N$. Then set $\hat{\xi}_{k-1}^{i}=$ $\xi_{k-1}^{I_{i}}$.

2. Mutation: For each $i=1, \cdots, N$, generate independently $N$ i.i.d. random variables $\left(\xi_{k, k}^{i}\right)_{0 \leq i \leq N}$ according to the transition kernel $M_{k}^{\prime}\left(\hat{\xi}_{k-1, k-1}^{i}, \cdot\right)$. Then set $\xi_{k}^{i}=\left(\hat{\xi}_{k-1}^{i}, \xi_{k, k}^{i}\right)$.

\subsection{Convergence analysis}

For general mean field particle interpretation models (5.3), several estimates can be derived for the above particle approximation model (see for instance [11]). For instance, for any $n \geq 0, r \geq 1$, and any $f_{n} \in \operatorname{Osc}_{1}\left(E_{n}\right)$, and any $N \geq 1$, we have the unbias and the mean error estimates:

$$
\begin{gathered}
\quad \mathbb{E}\left(\eta_{n}^{N}\left(f_{n}\right)\right)=\eta_{n}\left(f_{n}\right)=\mathbb{E}\left(\widehat{\eta}_{n}^{N}\left(f_{n}\right)\right) \\
\text { and } \quad \sqrt{N} \mathbb{E}\left(\left|\left[\eta_{n}^{N}-\eta_{n}\right]\left(f_{n}\right)\right|^{r}\right)^{\frac{1}{r}} \leq 2 a(r) \sum_{p=0}^{n} \beta\left(M_{p, n}\right),
\end{gathered}
$$

with the Dobrushin ergodic coefficients

$$
\beta\left(M_{p, n}\right):=\sup _{\left(x_{p}, y_{p} \in E_{p}\right)}\left\|M_{p, n}\left(x_{p}, .\right)-M_{p, n}\left(y_{p}, .\right)\right\|_{\mathrm{tv}}
$$

and the collection of constants $a(p)$ defined in (2.7). Arguing as in (2.8), for time homogeneous population sizes $N_{n}=N$, for any functions $f \in \operatorname{Osc}_{1}\left(E_{n}\right)$, 
we conclude that

$$
\begin{aligned}
& \mathbb{P}\left(\left|\left[\eta_{n}^{N}-\eta_{n}\right](f)\right| \geq \frac{b(n)}{\sqrt{N}}+\epsilon\right) \leq \exp \left(-\frac{N \epsilon^{2}}{2 b(n)^{2}}\right) \\
& \quad \text { with } b(n):=2 \sum_{p=0}^{n} \beta\left(M_{p, n}\right) .
\end{aligned}
$$

For the path space models (5.1), we have $\beta\left(M_{p, n}\right)=1$ so that the estimates (5.5) and (5.6) takes the form

$$
\sqrt{N} \mathbb{E}\left(\left|\left[\eta_{n}^{N}-\eta_{n}\right]\left(f_{n}\right)\right|^{r}\right)^{\frac{1}{r}} \leq 2 a(r)(n+1)
$$

and

$$
\mathbb{P}\left(\left|\left[\eta_{n}^{N}-\eta_{n}\right](f)\right| \geq \frac{2(n+1)}{\sqrt{N}}+\epsilon\right) \leq \exp \left(-\frac{N \epsilon^{2}}{8(n+1)^{2}}\right) .
$$

In the next lemma we extend these estimates to unbounded functions.

Lemma 5.1 For any $p \geq 1$, we denote by $p^{\prime}$ the smallest even integer greater than $p$. In this notation, for any $k \geq 0$ and any function $f$, we have the almost sure estimate

$$
\begin{aligned}
\sqrt{N} \mathbb{E}\left(\mid\left[\eta_{n}^{N}-\right.\right. & \left.\left.\eta_{k-1}^{N} M_{k-1, n}\right]\left.(f)\right|^{p} \mid \mathcal{F}_{k-1}^{N}\right)^{\frac{1}{p}} \\
& \leq 2 a(p) \sum_{l=k}^{n}\left[\eta_{k-1}^{N} M_{k-1, l}\left(\left|M_{l, n}(f)\right|^{p^{\prime}}\right)\right]^{\frac{1}{p^{\prime}}}
\end{aligned}
$$

In particular, for any $f \in \mathbb{L}_{p^{\prime}}\left(\eta_{n}\right)$, we have the non asymptotic estimates

$$
\sqrt{N} \mathbb{E}\left(\left|\left[\eta_{n}^{N}-\eta_{n}\right](f)\right|^{p}\right)^{1 / p} \leq 2 a(p)\|f\|_{p^{\prime}, \eta_{n}}(n+1) .
$$

\section{Proof:}

In writing $\eta_{-1}^{N} M_{0}=\eta_{0}$, for any $k \geq 0$ we have the decomposition

$$
\left[\eta_{n}^{N}-\eta_{k-1}^{N} M_{k, n}\right]=\sum_{l=k}^{n}\left[\eta_{l}^{N}-\left(\eta_{l-1}^{N} M_{l}\right)\right] M_{l, n}
$$

with the semigroup

$$
M_{k, n}=M_{k+1} M_{k+2} \ldots M_{n} .
$$

Using the fact that

$$
\mathbb{E}\left(\eta_{l}^{N}(f) \mid \eta_{l-1}^{N}\right)=\left(\eta_{l-1}^{N} M_{l}\right)(f)
$$

we prove that

$$
\mathbb{E}\left(\left|\left[\eta_{l}^{N}-\left(\eta_{l-1}^{N} M_{l}\right)\right](f)\right|^{p} \mid \mathcal{F}_{l-1}^{N}\right)^{\frac{1}{p}} \leq \mathbb{E}\left(\left|\left[\eta_{l}^{N}-\mu_{l}^{N}\right](f)\right|^{p} \mid \mathcal{F}_{l-1}^{N}\right)^{\frac{1}{p}},
$$


where $\mu_{l}^{N}:=\frac{1}{N} \sum_{i=1}^{N} \delta_{\zeta_{l}^{i}}$ stands for a independent copy of $\eta_{l}^{N}$ given $\eta_{l-1}^{N}$. Using Kintchine's type inequalities we have

$$
\begin{aligned}
\sqrt{N} \mathbb{E}\left(\left|\left[\eta_{l}^{N}-\mu_{l}^{N}\right](f)\right|^{p} \mid \mathcal{F}_{l-1}^{N}\right)^{\frac{1}{p}} & \leq 2 a(p) \mathbb{E}\left(\left|f\left(\xi_{l}^{1}\right)\right|^{p^{\prime}} \mid \mathcal{F}_{l-1}^{N}\right)^{\frac{1}{p^{\prime}}} \\
& =2 a(p)\left[\eta_{l-1}^{N} M_{l}\left(|f|^{p^{\prime}}\right)\right]^{\frac{1}{p^{\prime}}}
\end{aligned}
$$

Using the unbias property of the particle scheme, we have

$$
\forall k \leq l \leq n \quad \mathbb{E}\left(\eta_{l}^{N}(f) \mid \mathcal{F}_{k-1}^{N}\right)=\left(\eta_{k-1}^{N} M_{k-1, l}\right)(f)
$$

This implies that

$$
\begin{aligned}
\sqrt{N} \mathbb{E}\left(\left|\left[\eta_{l}^{N}-\left(\eta_{l-1}^{N} M_{l}\right)\right](f)\right|^{p} \mid \mathcal{F}_{k-1}^{N}\right)^{\frac{1}{p}} & \leq 2 a(p) \mathbb{E}\left(\eta_{l-1}^{N} M_{l}\left(|f|^{p^{\prime}}\right) \mid \mathcal{F}_{k-1}^{N}\right)^{\frac{1}{p^{\prime}}} \\
& =2 a(p)\left[\eta_{k-1}^{N} M_{k-1, l}\left(|f|^{p^{\prime}}\right)\right]^{\frac{1}{p^{\prime}}} .
\end{aligned}
$$

The end of the proof of (5.8) is now a direct application of Minkowski's inequality. The proof of (5.9) is a direct consequence of (5.8). This ends the proof of the lemma.

\subsection{Particle approximations of the Snell envelope}

In sub-section 5.1 we have presented a genealogical based algorithm whose occupation measures $\eta_{n}^{N}$ converge, as $N \uparrow \infty$, to the distribution $\eta_{n}$ of the reference Markov chain $\left(X_{0}^{\prime}, \ldots, X_{n}^{\prime}\right)$ from the origin, up to the final time horizon $n$. Mimicking formula (5.1), we define the particle approximation of the Markov transitions $M_{k}^{\prime}$ as follows :

$$
\widehat{M}_{k+1}^{\prime}(f)(x):=\frac{\eta_{n}^{N}\left(\left(1_{x} \circ \pi_{k}\right)\left(f \circ \pi_{k+1}\right)\right)}{\eta_{n}^{N}\left(\left(1_{x} \circ \pi_{k}\right)\right)}:=\frac{\sum_{1 \leq i \leq N} 1_{x}\left(\xi_{k, n}^{i}\right) f\left(\xi_{k+1, n}^{i}\right)}{\sum_{1 \leq i \leq N} 1_{x}\left(\xi_{k, n}^{i}\right)}
$$

for every state $x$ in the support $\widehat{E}_{k, n}$ of the measure $\eta_{n}^{N} \circ \pi_{k}^{-1}$. Notice that $\widehat{E}_{k, n}$ coincides with the collection of ancestors $\xi_{k, n}^{i}$ at level $k$ of the population of individuals at the final time horizon. This random set can alternatively be defined as the set of states $\xi_{k, k}^{i}$ of the particle population at time $k$ such that $\eta_{n}^{N}\left(\left(1_{\xi_{k, k}^{i}} \circ \pi_{k}\right)\right)>0$; more formally, we have

$$
\widehat{E}_{k, n}:=\cup_{1 \leq i \leq N}\left\{\xi_{k, k}^{i}: \eta_{n}^{N}\left(\left(1_{\xi_{k, k}^{i}} \circ \pi_{k}\right)\right)>0\right\}
$$

It is interesting to observe that the random Markov transitions $\widehat{M}_{k+1}^{\prime}$ coincides with the conditional distributions of the states $X_{k+1}^{\prime}$ given the current time states $X_{k}^{\prime}$ of a canonical Markov chain $X_{n}:=\left(X_{0}^{\prime}, \ldots, X_{n}^{\prime}\right)$ with distribution $\eta_{n}^{N}$ on the path space $E_{n}:=\left(E_{0}^{\prime} \times \ldots \times E_{n}^{\prime}\right)$. Thus, the flow of $k$-th time marginal measures

$$
\eta_{k, n}^{N}:=\frac{1}{N} \sum_{i=1}^{N} \delta_{\xi_{k, n}^{i}}
$$

$\mathrm{RR} \mathrm{n}^{\circ} 7303$ 
are connected by the following formula

$$
\forall k \leq l \leq n \quad \eta_{k, n}^{N} \widehat{M}_{k, l}^{\prime}=\eta_{l, n}^{N}
$$

with the semigroup $\widehat{M}_{k, l}^{\prime}$ associated with the Markov transitions $\widehat{M}_{k+1}^{\prime}$ and given by

$$
\widehat{M}_{k, l}^{\prime}(f)(x)=\widehat{M}_{k+1}^{\prime} \widehat{M}_{k+1}^{\prime} \ldots \widehat{M}_{l}^{\prime}(f)(x)=\frac{\eta_{n}^{N}\left(\left(1_{x} \circ \pi_{k}\right)\left(f \circ \pi_{l}\right)\right)}{\eta_{n}^{N}\left(\left(1_{x} \circ \pi_{k}\right)\right)}
$$

for every state $x$ in $\widehat{E}_{k, n}$. In connection with (5.10), we also have the following formula

$$
\eta_{k, n}^{N}=\frac{1}{N} \sum_{i=1}^{N}\left(N \eta_{n}^{N}\left(1_{\xi_{k, k}^{i}} \circ \pi_{k}\right)\right) \delta_{\xi_{k, k}^{i}}=\sum_{i=1}^{N} \eta_{n}^{N}\left(1_{\xi_{k, k}^{i}} \circ \pi_{k}\right) \delta_{\xi_{k, k}^{i}},
$$

with the proportion $\eta_{n}^{N}\left(1_{\xi_{k, k}^{i}} \circ \pi_{k}\right)$ of individuals at the final time horizon having the common ancestor $\xi_{k, k}^{i}$ at level $k$. It is also interesting to observe that

$$
\begin{aligned}
\mathbb{E}\left(\eta_{k, n}^{N}(f) \mid \mathcal{F}_{k}^{N}\right) & =\sum_{i=1}^{N} \mathbb{E}\left(\eta_{n}^{N}\left(1_{\xi_{k, k}^{i}} \circ \pi_{k}\right) \mid \mathcal{F}_{k}^{N}\right) f\left(\xi_{k, k}^{i}\right) \\
& =\sum_{i=1}^{N} \underbrace{\eta_{k}^{N} M_{k, n}\left(1_{\xi_{k, k}^{i}} \circ \pi_{k}\right)}_{=1 / N} f\left(\xi_{k, k}^{i}\right)=\eta_{k}^{N}(f)
\end{aligned}
$$

The Snell envelope associated with this particle approximation model is defined by the backward recursion:

$$
\widehat{u}_{k}(x)= \begin{cases}f_{k}(x) \vee \widehat{M}_{k+1}^{\prime}\left(u_{k+1}\right)(x) & \forall x \in \widehat{E}_{k, n} \\ 0 & \text { otherwise } .\end{cases}
$$

In terms of the ancestors at level $k$, this recursion takes the following form

$$
\forall 1 \leq i \leq N \quad \widehat{u}_{k}\left(\xi_{k, n}^{i}\right)=f_{k}\left(\xi_{k, n}^{i}\right) \vee \widehat{M}_{k+1}^{\prime}\left(\widehat{u}_{k+1}\right)\left(\xi_{k, n}^{i}\right) .
$$

In the sequel, the computation of the Snell envelope approximation $\left(\hat{u}_{k}\right)_{0 \leq k \leq n}$ will be called the Backward step and is summarized in the following algorithm.

\subsubsection{Backward algorithm}

Initialization At time step $k=n$, for all $i=1, \cdots, N$, set $\hat{u}_{n}\left(\xi_{n, n}^{i}\right)=f\left(\xi_{n, n}^{i}\right)$.

At each time step $k=n-1, \cdots, 0$, for all $i=1, \cdots, N$ set

$$
\hat{u}_{k}\left(\xi_{k, n}^{i}\right)=\frac{\sum_{j=1}^{N} \hat{u}_{k+1}\left(\xi_{k+1, n}^{j}\right) 1_{\xi_{k, n}^{j}=\xi_{k, n}^{i}}}{\sum_{j=1}^{N} 1_{\xi_{k, n}^{j}}} .
$$

For later use in the further development of this section, we quote a couple of technical lemmas. The first one provides some $\mathbb{L}_{p}$ estimates of the normalizing quantities of the Markov transitions $\widehat{M}_{k+1}^{\prime}$. The second one allows to quantify the deviations of $\widehat{M}_{k+1}^{\prime}$ around its limiting values $M_{k+1}^{\prime}$, as $N \rightarrow \infty$. 
Lemma 5.2 For any $p \geq 1$, and $0 \leq i \leq N$ we have the following uniform estimate

$$
\sup _{N \geq 1} \sup _{0 \leq l \leq k \leq n}\left\|\eta_{k}^{N}\left(1_{\xi_{l, k}^{i}} \circ \pi_{l}\right)^{-1}\right\|_{\mathbb{L}_{p}}<\infty .
$$

Lemma 5.3 For any $p \geq 1$, and $0 \leq i \leq N$ we have the following uniform estimate

$$
\sup _{0 \leq l \leq n}\left\|\widehat{M}_{l+1}^{\prime}(f)\left(\xi_{l, n}^{i}\right)-M_{l+1}^{\prime}(f)\left(\xi_{l, n}^{i}\right)\right\|_{\mathbb{L}_{p}} \leq c_{p}(n) / \sqrt{N},
$$

with some collection of finite constants $c_{p}(n)<\infty$ whose values only depend on the parameters $p$ and $n$.

The proofs of these lemmas are rather technical, thus there are postponed to the appendix.

We are now in position to state and prove the main result of this section.

Theorem 5.4 For any $p \geq 1$, and $0 \leq i \leq N$ we have the following uniform estimate

$$
\sup _{0 \leq k \leq n}\left\|\left(u_{k}-\widehat{u}_{k}\right)\left(\xi_{k, n}^{i}\right)\right\|_{\mathbb{L}_{p}} \leq c_{p}(n) / \sqrt{N}
$$

with some collection of finite constants $c_{p}(n)<\infty$ whose values only depend on the parameters $p$ and $n$.

Proof:

Firstly, we use the following decomposition

$$
\left|u_{k}-\widehat{u}_{k}\right| 1_{\widehat{E}_{k, n}} \leq \sum_{k \leq l \leq n-1} \widehat{M}_{k, l}^{\prime}\left|\left(\widehat{M}_{l+1}^{\prime}-M_{l+1}^{\prime}\right)\left(u_{l+1}\right)\right| 1_{\widehat{E}_{k, n}}
$$

. By construction, we have

$$
\widehat{M}_{k, l}^{\prime}\left|\left(\widehat{M}_{l+1}^{\prime}-M_{l+1}^{\prime}\right)\left(u_{l+1}\right)\right| 1_{\widehat{E}_{(k, n)}}=\widehat{M}_{k, l}^{\prime}\left|1_{\widehat{E}_{l, n}}\left(\widehat{M}_{l+1}^{\prime}-M_{l+1}^{\prime}\right)\left(u_{l+1}\right)\right| 1_{\widehat{E}_{k, n}} \text {. }
$$

By (5.11), if we set

$$
\widetilde{u}_{l+1}=\left|\left(\widehat{M}_{l+1}^{\prime}-M_{l+1}^{\prime}\right)\left(u_{l+1}\right)\right|
$$

on the set $\widehat{E}_{l, n}$, then we have that

$$
\widehat{M}_{k, l}^{\prime}\left(\widetilde{u}_{l+1}\right)\left(\xi_{k, n}^{i}\right)=\frac{\eta_{n}^{N}\left(\left(1_{\xi_{k, n}^{i}} \circ \pi_{k}\right)\left(\widetilde{u}_{l+1} \circ \pi_{l}\right)\right)}{\eta_{n}^{N}\left(\left(1_{\xi_{k, n}^{i}} \circ \pi_{k}\right)\right)} .
$$

For any $p \geq 1$, we have

$$
\begin{aligned}
&\left\|\widehat{M}_{k, l}^{\prime}\left(\widetilde{u}_{l+1}\right)\left(\xi_{k, n}^{i}\right)\right\|_{\mathbb{L}_{p}} \leq\left\|\eta_{n}^{N}\left(\left(1_{\xi_{k, n}^{i}} \circ \pi_{k}\right)\right)^{-1}\right\|_{\mathbb{L}_{2}}^{1 / p} \\
& \times \mathbb{E}\left(\eta_{n}^{N}\left(\left(1_{\xi_{k, n}^{i}} \circ \pi_{k}\right)\left(\widetilde{u}_{l+1} \circ \pi_{l}\right)^{2 p}\right)\right)^{1 /(2 p)} .
\end{aligned}
$$

This implies that

$$
\left\|\widehat{M}_{k, l}^{\prime}\left(\widetilde{u}_{l+1}\right)\left(\xi_{k, n}^{i}\right)\right\|_{\mathbb{L}_{p}} \leq\left\|\eta_{n}^{N}\left(\left(1_{\xi_{k, n}^{i}} \circ \pi_{k}\right)\right)^{-1}\right\|_{\mathbb{L}_{2}}^{1 / p} \times \sup _{1 \leq j \leq N}\left\|\widetilde{u}_{l+1}\left(\xi_{l, n}^{j}\right)\right\|_{\mathbb{L}_{2 p}} .
$$

The proof of (5.14) is now a clear consequence of Lemma 5.2 and Lemma 5.3. This ends the proof of the theorem. 


\subsection{Bias analysis}

To end this subsection, we will prove that just as the bias of the BroadieGlasserman type estimators, the bias of the genealogical tree based estimator is always positive.

Notice that, for any $0 \leq k \leq n$, function $f$ on space $E_{k}^{\prime}$ and any $i \in$ $\{1, \ldots, N\}$ we have

$$
\mathbb{E}\left(f\left(\xi_{k+1, n}^{i}\right) \mid \xi_{k, n}\right)=M_{k+1} f\left(\xi_{k, n}^{i}\right) .
$$

This is because in the neutral genealogical tree model, the selection steps are independent of the mutations steps. Here, $\xi_{k, n}$ contains all the information on the construction of the tree plus the information on the values of the nodes on this tree at instant $\mathrm{k}$. The equation (5.16) comes from the fact that given the information $\xi_{k, n}$ the particle $\xi_{k+1, n}^{i}$ follows the distribution $M_{k+1}^{\prime}\left(\xi_{k, n}^{i}, \cdot\right)$.

Theorem 5.5 For any $0 \leq k \leq n$ and any $i \in\{1, \ldots, N\}$, we have

$$
\mathbb{E}\left(\widehat{u}_{k}\left(\xi_{k, n}^{i}\right) \mid \xi_{k, n}\right) \geq u_{k}\left(\xi_{k, n}^{i}\right) \text {. }
$$

\section{Proof:}

To prove this, we will use a simple induction argument.

For $l=n, \widehat{u}_{n}=u_{n}$, then we easily check that the following inequality is verified for all $i=1, \ldots, N$,

$$
\mathbb{E}\left(\widehat{u}_{l}\left(\xi_{l, n}^{i}\right) \mid \xi_{l, n}\right) \geq u_{l}\left(\xi_{l, n}^{i}\right) .
$$

Assume that (5.18) is verified for all $i=1, \ldots, N$ and let us prove that the same inequality is valid for instant $l-1$.

With the elementary decomposition:

$$
\begin{aligned}
\mathbb{E}\left(\widehat{M}_{l}^{\prime}\left(\widehat{u}_{l}\right)\left(\xi_{l-1, n}^{i}\right) \mid \xi_{l-1, n}\right) & =\mathbb{E}\left(\frac{\sum_{j=1}^{N} \widehat{u}_{l}\left(\xi_{l, n}^{j}\right) 1_{\xi_{l-1, n}^{j}}=\xi_{l-1, n}^{i}}{\sum_{j=1}^{N} 1_{\xi_{l-1, n}^{j}}=\xi_{l-1, n}^{i}} \mid \xi_{l-1, n}\right) \\
& =\frac{\sum_{j=1}^{N} \mathbb{E}\left(\widehat{u}_{l}\left(\xi_{l, n}^{j}\right) \mid \xi_{l-1, n}\right) 1_{\xi_{l-1, n}^{j}}=\xi_{l-1, n}^{i}}{\sum_{j=1}^{N} 1_{\xi_{l-1, n}^{j}}=\xi_{l-1, n}^{i}} .
\end{aligned}
$$

By assumption (5.18) and equation (5.16), we have

$$
\begin{aligned}
\mathbb{E}\left(\widehat{u}_{l}\left(\xi_{l, n}^{j}\right) \mid \xi_{l-1, n}\right) & \geq \mathbb{E}\left(u_{l}\left(\xi_{l, n}^{j}\right) \mid \xi_{l-1, n}\right) \\
& =M_{l} u_{l}\left(\xi_{l-1, n}^{j}\right) .
\end{aligned}
$$

Applying the precedent decomposition, it follows easily

$$
\begin{aligned}
\mathbb{E}\left(\widehat{M}_{l} \widehat{u}_{l}\left(\xi_{l-1, n}^{i}\right) \mid \xi_{l-1, n}\right) & \geq \frac{\sum_{j=1}^{N} M_{l} u_{l}\left(\xi_{l-1, n}^{i}\right) 1_{\xi_{l-1, n}^{j}=\xi_{l-1, n}^{i}}}{\sum_{j=1}^{N} 1_{\xi_{l-1, n}^{j}}=\xi_{l-1, n}^{i}} \\
& =M_{l} u_{l}\left(\xi_{l-1, n}^{i}\right)
\end{aligned}
$$

Then we can end this proof by Jensen's inequality

$$
\begin{aligned}
\mathbb{E}\left(\widehat{u}_{l-1}\left(\xi_{l-1, n}^{i}\right) \mid \xi_{l-1, n}\right) & \geq f_{l-1}\left(\xi_{l-1, n}^{i}\right) \vee \mathbb{E}\left(\widehat{M}_{l} \widehat{u}_{l}\left(\xi_{l-1, n}^{i}\right) \mid \xi_{l-1, n}\right) \\
& \geq f_{l-1}\left(\xi_{l-1, n}^{i}\right) \vee M_{l} u_{l}\left(\xi_{l-1, n}^{i}\right) \\
& =u_{l-1}\left(\xi_{l-1, n}^{i}\right) .
\end{aligned}
$$




\subsection{Numerical simulations}

In this section, we give numerical examples to test the genealogical tree algorithm on two types of options from dimension 1 up to 6 .

\subsubsection{Prices dynamics and options model}

Our numerical examples are taken from Bouchard and Warin [7] who have provided precise approximations of option values in their examples. The asset prices are modelized by a $d$-dimensional Markov process $\left(\tilde{X}_{t}\right)$ such that each component (i.e. each asset) follows a geometric Brownian motion under the risk-neutral measure, that is, for assets $i=1, \cdots, d$,

$$
\frac{d \tilde{X}_{t}(i)}{\tilde{X}_{t}(i)}=r d t+\sigma_{i} d z_{t}^{i}
$$

where $z^{i}$, for $i=1, \cdots, d$ are independent standard Brownian motions. The interest rate $r$ is set to $5 \%$ annually. We also assume that for all $i=1, \cdots, d$, $\tilde{X}_{t_{0}}(i)=1$ and $\sigma_{i}=20 \%$ annually.

We consider two different Bermudan options with maturity $T=1$ year and 11 equally distributed exercise opportunities at dates $t_{k}=k T / n$ with $k=$ $0,1, \cdots, n=10$, associated with two different payoffs:

1. a geometric average put option with strike $K=1$ and payoff ( $K-$ $\left.\prod_{i=1}^{d} \tilde{X}_{T}(i)\right)_{+}$

2. an arithmetic average put option with strike $K=1$ and payoff $(K-$ $\left.\frac{1}{d} \sum_{i=1}^{d} \tilde{X}_{T}(i)\right)_{+}$.

Note that the geometric average put payoff involves the process $\prod_{i=1}^{d} \tilde{X}(i)$ which can be identified to a one-dimensional non standard exponential Brownian motion. This trick was used, in 7], to compute a precise benchmark option value by PDE technics. We report on Figure 1 the benchmark option values computed in [7], for both the geometric and arithmetic put options (by using respectively the one dimensional PDE method and the Longstaff-Schwartz method with $3 \times 10^{7}$ simulations).

\begin{tabular}{|c||c|c|c|c|c|c|}
\hline Number of assets & 1 & 2 & 3 & 4 & 5 & 6 \\
\hline \hline Geometric Payoff & 0.06033 & 0.07815 & 0.08975 & 0.09837 & 0.10511 & 0.11073 \\
\hline Arithmetic Payoff & 0.06033 & 0.03881 & 0.02945 & 0.02403 & 0.02070 & 0.01895 \\
\hline
\end{tabular}

Figure 1: Benchmark values for the geometric and arithmetic put options (taken from [7]). 


\subsubsection{State space discretization}

The genealogical tree algorithm is designed for finite state spaces. Hence, before applying it to the aforementioned continuous space examples, we have to approximate the continuous state space Markov chain solution of (5.19) by a Markov chain with a finite state space. To this end, one can first discretize the state space using either a random tree, or a stochastic mesh, or a Binomial tree or a quantization approach ... In our numerical simulations, the quantization discretization seemed to be the most efficient.

State space partitioning Here, we propose to use a quantization-like approach for the space discretization step. We simulate a first set of $M$ iid paths at each $n+1$ possible exercise dates $t_{0}, \cdots, t_{n},\left(\tilde{X}_{t_{k}}^{i}\right)_{k=0, \cdots, n}^{i=1, \cdots, M}$ according to dynamic (5.19). Assume now, that it exists two integers $N^{\prime}$ and $P$ such that $M$ can be written as the product $M=N^{\prime} P$. Then, at each time step $t_{k}$, the particle set $\mathcal{S}_{k}=\left\{\tilde{X}_{t_{k}}^{1}, \cdots, \tilde{X}_{t_{k}}^{M}\right\}$ can be partitioned into $N^{\prime}$ localized subsets $\left\{\mathcal{S}_{k}^{1}, \cdots, \mathcal{S}_{k}^{N^{\prime}}\right\}$ of $P$ particles. Assume now that it exists $d$ integers $\left(Q_{1}, \cdots, Q_{d}\right)$ such that $N^{\prime}$ can be written as the product $N^{\prime}=Q_{1} \cdots Q_{d}$. Assume for simplicity that $N^{\prime}=Q^{d}$. One way to build this partition $\left\{\mathcal{S}_{k}^{1}, \cdots, \mathcal{S}_{k}^{N^{\prime}}\right\}$ is then apply the following procedure as in [7]:

1. sort the particles according to the first coordinate and split the sorted particles into $Q$ subsets containing the same number of particles $Q^{d-1} P$;

2. if $d \geq 2$, for each subset, sort the particles according to the second coordinate and split the sorted particles into $Q$ subsets containing the same number of particles $Q^{d-2} P$, which finally leads to $Q^{2}$ subsets containing the same number of particles $Q^{d-2} P$;

3. if $d \geq 3$, repeat this procedure recursively, in each direction $i=3, \cdots, d$.

This operation is realized with a complexity $O(d M \log (M))$ and produces a partitions of $\mathcal{S}_{k}$ into $N^{\prime}=Q^{d}$ subsets $\mathcal{S}_{k}^{1}, \cdots, \mathcal{S}_{k}^{N^{\prime}}$ with the same number $P$ of particles.

Now, for each subset $\mathcal{S}_{k}^{j}$, for $j=1, \cdots, N^{\prime}$, we compute a representative state, $S_{k}^{j}$ as the average particle over all the elements of $\mathcal{S}_{k}^{j}$. Then at each time step $t_{k}$ for $k=1, \cdots, n$, we will consider the finite state space $E_{k}=\left\{S_{k}^{1}, \cdots, S_{k}^{N^{\prime}}\right\}$ and we set $E_{0}=\left\{X_{t_{0}}\right\}$. In the sequel, the discrete points $S_{k}^{1}, \cdots, S_{k}^{N^{\prime}}$ will be referred to as the sites.

Finite state space Markov chain Assume now that a sequence of finite state spaces $E_{k} \subset \mathbb{R}^{d}$ is given for $k=1, \cdots, n$ (for instance by the above procedure). We define a finite state space Markov chain $\left(X_{k}^{\prime}\right)_{k=0, \cdots, n}$ such that $X_{0}^{\prime}=\tilde{X}_{t_{0}}$ and for all $k=1, \cdots, n$,

- $X_{k}^{\prime} \in E_{k}$;

- $\mathbb{P}\left(X_{k}^{\prime}=S_{k}^{j} \mid X_{k-1}^{\prime}=S_{k-1}^{i}\right)=\mathbb{P}\left(\tilde{X}_{t_{k}} \in V_{k}^{j} \mid \tilde{X}_{t_{k-1}}=S_{k-1}^{i}\right)$, where $V_{k}^{j}$ denotes the Voronoi cell associated to the site $S_{k}^{j}$ in the the discrete set $E_{k}$ and $\left(\tilde{X}_{t_{k}}\right)$ is the Markov process verifying (5.19) observed at the discrete times $t_{0}, \cdots, t_{n}$. 
To simulate a transition of the Markov Chain $\left(X_{k}^{\prime}\right)_{k=0, \cdots, n}$ from the state $S_{k-1}^{i} \in$ $E_{k-1}$ at the time step $k-1$ to the time step $k$ one can apply the following procedure:

1. simulate a random variable $\tilde{X}_{t_{k}}$ according to $\tilde{M}_{k}\left(S_{k-1}^{i}, \cdot\right)$ where $\tilde{M}_{k}$ denotes the transition kernel of the continuous state space Markov chain verifying (5.19) from time $t_{k-1}$ to $t_{k}$;

2. set $X_{k}^{\prime}=S_{k}^{i^{*}}$, where $S_{k}^{i^{*}}$ is the nearest neighbor of $\tilde{X}_{t_{k}}$ among the elements of $E_{k}$.

\subsubsection{Complexity}

In comparison with the quantization method proposed in 24, the genealogical algorithm based on the above space discretization only needs to simulate the finite state space Markov chain $\left(X_{k}^{\prime}\right)$ and avoids the time consuming computation of the transition probabilities.

In terms of complexity, the major part of the computing time is spent in the forward step discribed in subsection 5.1 .1 for simulating the discrete space Markov chain $\left(X_{k}^{\prime}\right)$. More precisely, for each transition, one has to compute a nearest neighbor among $N^{\prime}$ sites which finally leads to a complexity of order $O\left(N N^{\prime}\right)$ by time step, when considering the whole set of $N$ particles.

In terms of approximation error, one can decompose the error induced by the whole procedure, on the Snell envelope approximation, into the sum of two terms:

1. the state space discretization error which can be upper bounded, according to [24] or Proposition 3.6, by $\frac{c}{N^{\prime 1 / d}}$;

2. the error induced by the genealogical tree algorithm, which could be upper bounded, according to the proof of Theorem [5.4 by $c \frac{N^{\prime \beta}}{N^{1 / 2}}$, for a given positive real $\beta>0$.

Hence, to minimize the resulting upper bound on the global error, one has to choose judiciously the number of sites $N^{\prime}$ as a function of the number of particles such that $N^{\prime}=0\left(N^{\frac{d}{2 \beta d+2}}\right)$. With this choice, the complexity of the global procedure is of order $O\left(N^{\frac{(1+2 \beta) d+2}{2 \beta d+2}}\right)$, with an approximation error bounded by $\frac{c}{N^{\frac{1}{2 \beta d+2}}}$. In our numerical simulations, we have set $\beta=1 / 2$ so that the complexity grows with the dimension from $N^{4 / 3}, N^{3 / 2}, N^{8 / 5}, \cdots, N^{2}$ for dimensions $d=1,2,3, \cdots, \infty$.

On the other hand, in the backward step, (described in subsection 5.3.1) consisting of computing the Snell envelope, our algorithm only requires a complexity which is linear in the number of particles, $N$. Hence, for a given underlying price process, our approach can rapidly approximate several Bermudan options with different payoff functions.

\subsubsection{Numerical results}

For each example, we have performed the algorithm for different numbers of particles for $N=5 \times 10^{3}, 1 \times 10^{4}, 2.5 \times 10^{4}, 5 \times 10^{4}, 1 \times 10^{5}, 2 \times 10^{5}, 4 \times$ $10^{5}, 1 \times 10^{6}, 2 \times 10^{6}$. In each case, the sites were computed on the base of 
$M=\max \left(500000,50 \times N^{\prime}\right)=\max \left(500000,50 \times N^{\frac{d}{d+2}}\right)$ simulations. Many runs of the algorithm were performed to build box plots for our estimates: 50 runs for $N<10^{6}$ and 24 runs for $N=1 \times 10^{6}$ and $N=2 \times 10^{6}$. Simulations results are reported on Figure 2 for the geometric put payoff and on Figure 3 for the arithmetic put payoff. If we compare our numerical results with those reported in [7, for the Longstaff-Schwartz, Malliavin and quantization algorithms, it seems that our algorithm performs well and can give better performances, even for dimension $d=6$. The empirical convergence rate seems to be faster than the upper bound of $\frac{c}{N^{\frac{1}{d+2}}}$.

Looking into further applications, this algorithm is also well suited for Bermudan options with path dependent payoff. Indeed, by construction, the genealogical tree algorithm is defined in terms of the historical process, then it is able to compute conditional expectations w.r.t. the whole past of the process with no additional complexity.

In the same vein, we believe that this algorithm and the related convergence result could be extended, with slight modifications, to the more general case of reflected Backward Stochastic Differential Equations (BSDE) with non zero driver that does not depend on the $z$ variable and which satisfies suitable regularity conditions.

Finally, in further research, it could also be interesting to extend this algorithm for the computation of price sensitivities for hedging purposes. 


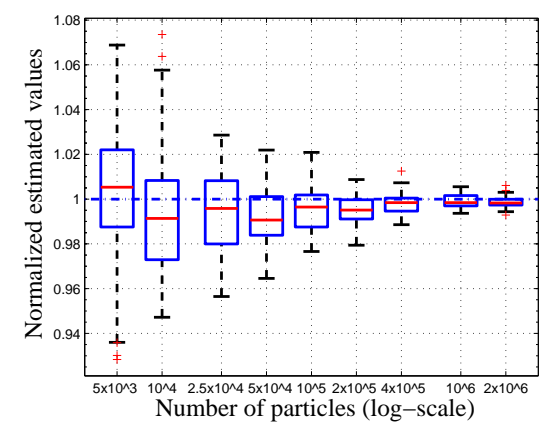

(a) $d=1$

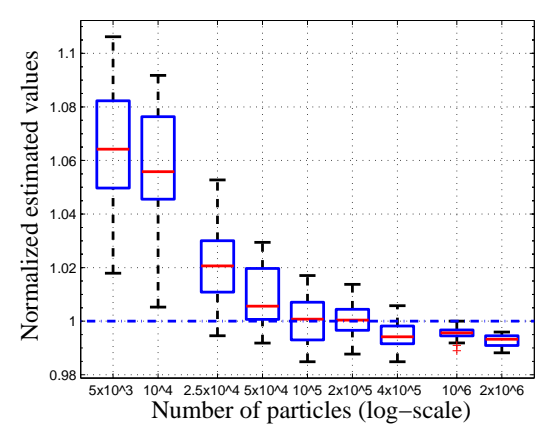

(c) $d=3$

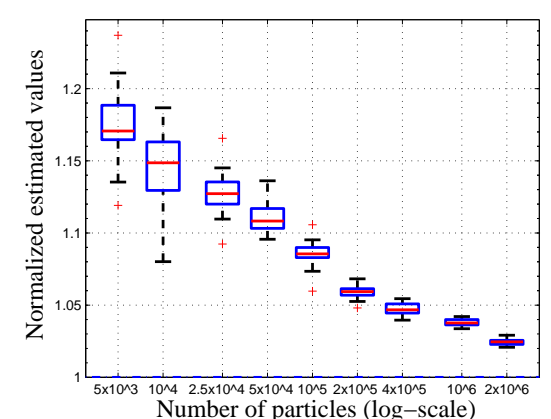

(e) $d=5$

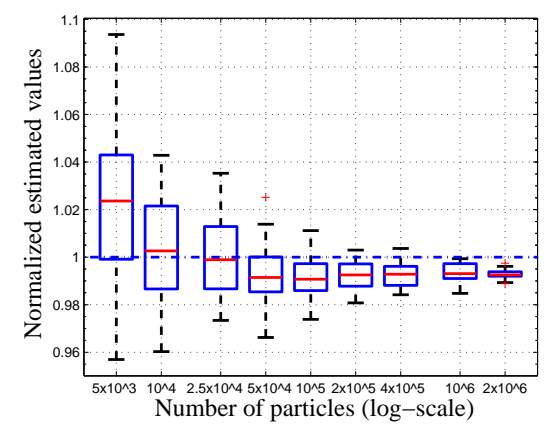

(b) $d=2$

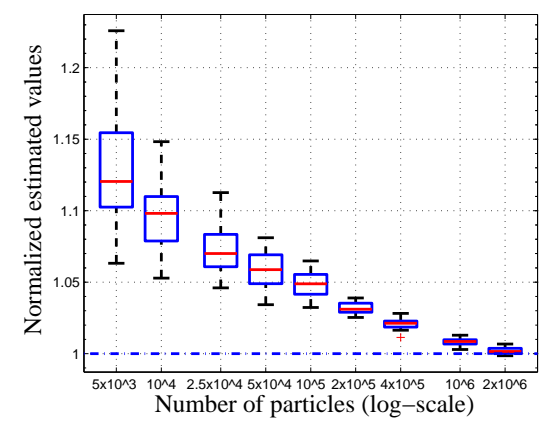

(d) $d=4$

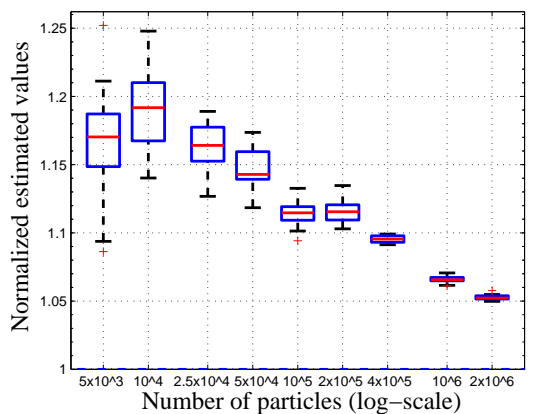

(f) $d=6$

Figure 2: Boxplots for estimated option values (divided by the benchmark values) as a function of the number of particles for the geometric put-payoff. The box stretches from the 25 th percentile to the 75 th percentile, the median is shown as a line across the box, the whiskers extend from the box out to the most extreme data value within 1.5 IQR (Interquartile Range) and red crosses indicates outliers. 


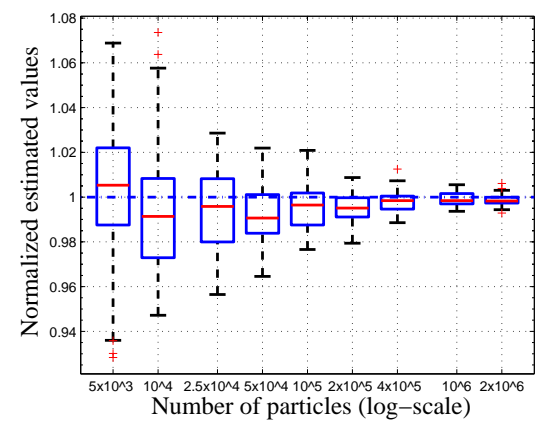

(a) $d=1$

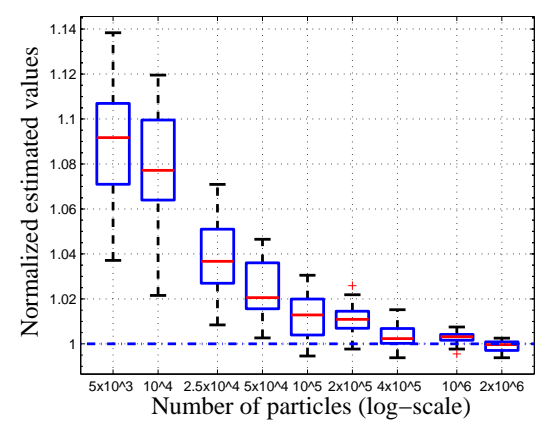

(c) $d=3$

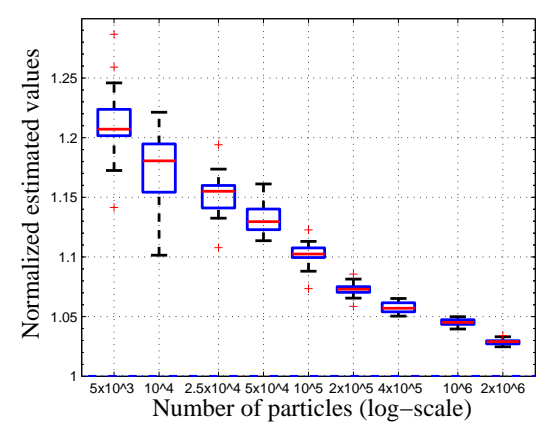

(e) $d=5$

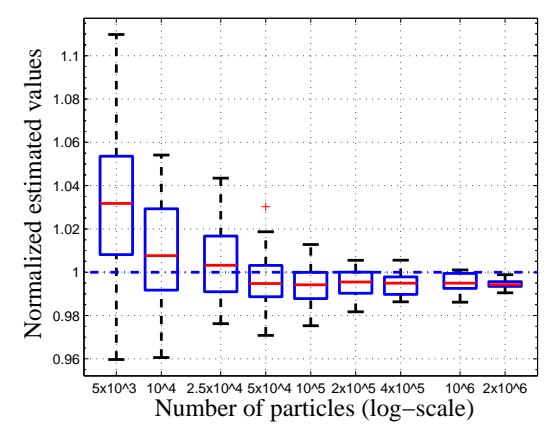

(b) $d=2$

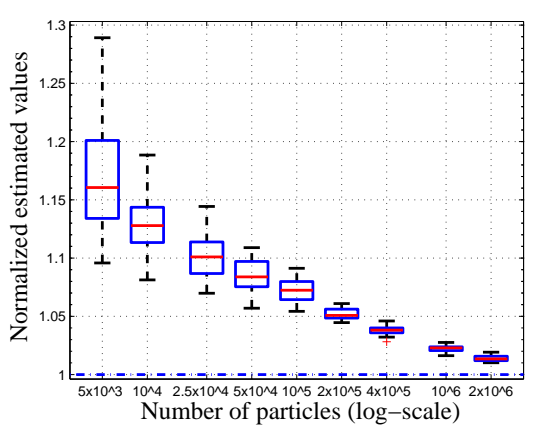

(d) $d=4$

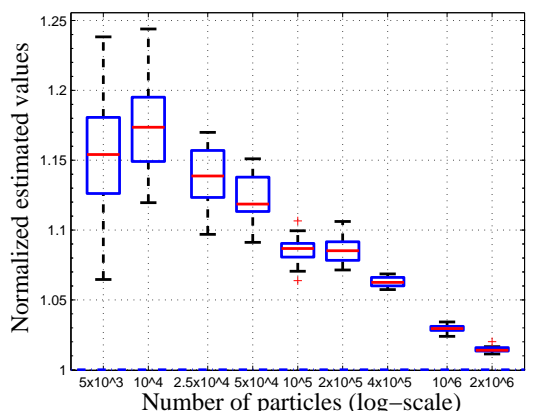

(f) $d=6$

Figure 3: Boxplots for estimated option values (divided by the benchmark values) as a function of the number of particles for the arithmetic put-payoff. The box stretches from the 25 th percentile to the 75 th percentile, the median is shown as a line across the box, the whiskers extend from the box out to the most extreme data value within 1.5 IQR (Interquartile Range) and red crosses indicates outliers.

\section{Appendix}

\subsection{Proof of Lemma 5.2;}

We set

$$
\delta_{l, n}(N):=\inf _{x \in E_{l}^{\prime}} \eta_{n}^{N}\left(g_{l, x}\right)
$$


with the function $g_{l, x}$ defined in (6.3), and we notice that

$$
\mathbb{P}\left(\delta_{l, n}(N)=0\right) \leq \sum_{x \in E_{l}^{\prime}} \mathbb{P}\left(\eta_{n}^{N}\left(g_{l, x}\right)=0\right)
$$

On the other hand, for any $\epsilon \in[0,1)$ we have

$$
\mathbb{P}\left(\eta_{n}^{N}\left(g_{l, x}\right)=0\right) \leq \mathbb{P}\left(\left|\eta_{n}^{N}\left(g_{l, x}\right)-\eta_{n}\left(g_{l, x}\right)\right|>\epsilon \eta_{n}\left(g_{l, x}\right)\right) .
$$

Arguing as in (5.7), for any $x \in E_{l}^{\prime}$ s.t. $\eta_{n}\left(g_{l, x}\right)\left(=\mathbb{P}\left(X_{l}^{\prime}=x\right)\right)>0$ we prove that

$$
\sqrt{N} \mathbb{E}\left(\left|\eta_{n}^{N}\left(g_{l, x}\right)-\eta_{n}\left(g_{l, x}\right)\right|^{r}\right)^{\frac{1}{r}} \leq 2 a(r)(n+1) \eta_{n}\left(g_{l, x}\right)^{-1}
$$

and therefore

$$
\mathbb{P}\left(\left|\eta_{n}^{N}\left(g_{l, x}\right)-\eta_{n}\left(g_{l, x}\right)\right| \geq\left(\frac{2(n+1)}{\sqrt{N}}+\epsilon\right) \eta_{n}\left(g_{l, x}\right)\right) \leq \exp \left(-\frac{N \epsilon^{2}}{8(n+1)^{2}}\right) .
$$

For any $N \geq(2(n+1) /(1-\epsilon))^{2}$, this implies that

$$
\mathbb{P}\left(\delta_{l, n}(N)=0\right) \leq \operatorname{Card}\left(E_{l}^{\prime}\right) \exp \left(-\frac{N \epsilon^{2}}{8(n+1)^{2}}\right) .
$$

If we choose, $\epsilon=1 / 2$ and $N \geq(4(n+1))^{2}$, we conclude that

$$
\mathbb{P}\left(\delta_{l, n}(N)=0\right) \leq \operatorname{Card}\left(E_{l}^{\prime}\right) \exp \left(-\frac{N}{32(n+1)^{2}}\right) .
$$

On the other hand, by construction we have the almost sure estimate

$$
\eta_{n}^{N}\left(g_{l, \xi_{l, n}^{i}}\right)=\sum_{x \in E_{l}^{\prime}} \eta_{n}^{N}\left(g_{l, x}\right) 1_{\xi_{l, n}^{i}=x} \geq \delta_{l, n}(N) 1_{\delta_{l, n}(N)>0}+\frac{1}{N} 1_{\delta_{l, n}(N)=0}
$$

from which we find that

$$
\eta_{n}^{N}\left(g_{l, \xi_{l, n}^{i}}\right)^{-1} \leq \delta_{l, n}(N)^{-1} 1_{\delta_{l, n}(N)>0}+N 1_{\delta_{l, n}(N)=0}
$$

Therefore, we have

$$
\begin{aligned}
\left\|\eta_{n}^{N}\left(g_{l, \xi_{l, n}^{i}}\right)^{-1}\right\|_{\mathbb{L}_{p}} & \leq\left\|\delta_{l, n}(N)^{-1} 1_{\delta_{l, n}(N)>0}\right\|_{\mathbb{L}_{p}}+N\left\|1_{\delta_{l, n}(N)=0}\right\|_{\mathbb{L}_{p}} \\
& \leq \sum_{x \in E_{l}^{\prime}}\left\|\eta_{n}^{N}\left(g_{l, x}\right)^{-1} 1_{\eta_{n}^{N}\left(g_{l, x}\right)>0}\right\| \mathbb{L}_{p}+N \mathbb{P}\left(\delta_{l, n}(N)=0\right)^{1 / p} .
\end{aligned}
$$

If we set $\bar{g}_{l, n}(x)=g_{l, x} / \eta_{n}\left(g_{l, x}\right)$, using the fact that

$$
\frac{1}{1-u}=1+u+u^{2}+\frac{u^{3}}{1-u}
$$

for any $u \neq 1$, and $\eta_{n}^{N}\left(\bar{g}_{l, x}\right)^{-1} 1_{\eta_{n}^{N}\left(g_{l, x}\right)>0} \leq N \eta_{n}\left(g_{l, x}\right)$, we find that

$$
\begin{aligned}
\eta_{n}^{N}\left(\bar{g}_{l, x}\right)^{-1} 1_{\eta_{n}^{N}\left(g_{l, x}\right)>0} \leq 1+\mid 1- & \eta_{n}^{N}\left(\bar{g}_{l, x}\right) \mid+\left(1-\eta_{n}^{N}\left(\bar{g}_{l, x}\right)\right)^{2} \\
& +N \eta_{n}\left(g_{l, x}\right)\left|1-\eta_{n}^{N}\left(\bar{g}_{l, x}\right)\right|^{3} .
\end{aligned}
$$


Combining this estimate with (6.1), for any $p \geq 1$ we prove the following upper bound

$$
\begin{aligned}
\left\|\eta_{n}^{N}\left(\bar{g}_{l, x}\right)^{-1} 1_{\eta_{n}^{N}\left(g_{l, x}\right)>0}\right\|_{\mathbb{L}_{p}} \leq 1+\frac{1}{\sqrt{N}} 2 a(p)(n+1) & +(2 a(2 p)(n+1))^{2} \frac{1}{N} \\
& +\frac{1}{\sqrt{N}}(2 a(3 p)(n+1))^{3}
\end{aligned}
$$

from which we find the rather crude estimates

$$
\left\|\eta_{n}^{N}\left(\bar{g}_{l, x}\right)^{-1} 1_{\eta_{n}^{N}\left(g_{l, x}\right)>0}\right\|_{\mathbb{L}_{p}} \leq 1+\frac{3}{\sqrt{N}} a^{\prime}(p)(n+1)^{3}
$$

with the collection of finite constants $a^{\prime}(p):=2 a(p)+(2 a(2 p))^{2}+(2 a(3 p))^{3}$. Using the above exponential inequalities, we find that

$$
\begin{aligned}
& \left\|\eta_{n}^{N}\left(g_{l, \xi_{l, n}^{i}}\right)^{-1}\right\|_{\mathbb{L}_{p}} \\
& \leq \sum_{x \in E_{l}^{\prime}} \frac{1}{\eta_{n}\left(g_{l, x}\right)}\left[1+\frac{3}{\sqrt{N}} a^{\prime}(p)(n+1)^{3}\right]+N \operatorname{Card}\left(E_{l}^{\prime}\right)^{1 / p} \exp \left(-\frac{N}{32 p(n+1)^{2}}\right) .
\end{aligned}
$$

This ends the proof of the lemma.

\subsection{Proof of Lemma 5.3;}

By construction, we have

$$
\forall x \in \widehat{E}_{l, n} \quad M_{l+1}^{\prime}(f)(x)=\frac{\eta_{l}^{N} M_{l, n}\left(\left(1_{x} \circ \pi_{l}\right)\left(f \circ \pi_{l+1}\right)\right)}{\eta_{l}^{N} M_{l, n}\left(\left(1_{x} \circ \pi_{l}\right)\right)} .
$$

Thus, by (6.2) we have

$$
\widehat{M}_{l+1}^{\prime}(f)(x)-M_{l+1}^{\prime}(f)(x):=\frac{\eta_{n}^{N}\left(g_{l, x} f_{l+1}\right)}{\eta_{n}^{N}\left(g_{l, x}\right)}-\frac{\eta_{l}^{N} M_{l, n}\left(g_{l, x} f_{l+1}\right)}{\eta_{l}^{N} M_{l, n}\left(g_{l, x}\right)}
$$

for any $x \in \widehat{E}_{l, n}$, with the collection of functions

$$
g_{l, x}:=1_{x} \circ \pi_{l} \quad \text { and } \quad f_{l+1}:=f \circ \pi_{l+1} .
$$

It is readily checked that

$$
\widehat{M}_{l+1}^{\prime}(f)(x)-M_{l+1}^{\prime}(f)(x)=\frac{1}{\eta_{n}^{N}\left(\bar{g}_{l, x}^{N}\right)}\left[\eta_{n}^{N}\left(\bar{f}_{l+1, x}^{N}\right)-\eta_{l}^{N} M_{l, n}\left(\bar{f}_{l+1, x}^{N}\right)\right]
$$

for any $x \in \widehat{E}_{l, n}$, with the pair of $\mathcal{F}_{l}^{N}$-measurable functions

$\bar{f}_{l+1, x}^{N}:=\frac{g_{l, x}}{\eta_{l}^{N} M_{l, n}\left(g_{l, x}\right)}\left[f_{l+1}-\frac{\eta_{l}^{N} M_{l, n}\left(g_{l, x} f_{l+1}\right)}{\eta_{l}^{N} M_{l, n}\left(g_{l, x}\right)}\right] \quad$ and $\quad \bar{g}_{l, x}^{N}=\frac{g_{l, x}}{\eta_{l}^{N} M_{l, n}\left(g_{l, x}\right)}$.

It is also important to observe as $g_{l, x}$ varies only on $E_{l}^{\prime}$, then

$$
\eta_{l}^{N} M_{l, n}\left(g_{l, x}\right)=\eta_{l}^{N}\left(g_{l, x}\right) \leq 1
$$


In this notation, for any $0 \leq i \leq N$ and any $p \geq 1$, we have

$$
\begin{aligned}
& \left\|\widehat{M}_{l+1}^{\prime}(f)\left(\xi_{l, n}^{i}\right)-M_{l+1}^{\prime}(f)\left(\xi_{l, n}^{i}\right)\right\|_{\mathbb{L}_{p}} \\
& \quad \leq\left.\left\|\eta_{n}^{N}\left(g_{l, \xi_{l, n}^{i}}\right)^{-1}\right\|\right|_{\mathbb{L}_{2 p}}\left\|\eta_{n}^{N}\left(\bar{f}_{l+1, \xi_{l, n}^{i}}^{N}\right)-\eta_{l}^{N} M_{l, n}\left(\bar{f}_{l+1, \xi_{l, n}^{i}}^{N}\right)\right\|_{\mathbb{L}_{2 p}}
\end{aligned}
$$

The collection of random functions $\bar{f}_{l+1, \xi_{l, l}^{j}}^{N}$ are well defined and we have

$$
\begin{aligned}
& \left(\eta_{n}^{N}\left(\bar{f}_{l+1, \xi_{l, n}^{i}}^{N}\right)-\eta_{l}^{N} M_{l, n}\left(\bar{f}_{l+1, \xi_{l, n}^{i}}^{N}\right)\right)^{\beta} \\
& =\frac{1}{\eta_{l}^{N}\left(g_{l, \xi_{l, n}^{i}}\right)} \frac{1}{N} \sum_{j=1}^{N}\left[\eta_{n}^{N}\left(\bar{f}_{l+1, \xi_{l, l}^{j}}^{N}\right)-\eta_{l}^{N} M_{l, n}\left(\bar{f}_{l+1, \xi_{l, l}^{j}}^{N}\right)\right]^{\beta} 1_{\xi_{l, l}^{j}=\xi_{l, n}^{i}}
\end{aligned}
$$

for any $\beta \geq 0$. Combining the above formula for $\beta=2 p$ and Holder's inequality, we prove that

$$
\begin{aligned}
& \left\|\eta_{n}^{N}\left(\bar{f}_{l+1, \xi_{l, n}^{i}}^{N}\right)-\eta_{l}^{N} M_{l, n}\left(\bar{f}_{l+1, \xi_{l, n}^{i}}^{N}\right)\right\|_{\mathbb{L}_{2 p}} \\
& \leq\left\|\eta_{l}^{N}\left(g_{l, \xi_{l, n}^{i}}\right)^{-1}\right\|_{\mathbb{L}_{q}}^{1 /(2 p)} \times \sup _{1 \leq j \leq N}\left\|\eta_{n}^{N}\left(\bar{f}_{l+1, \xi_{l, l}^{j}}^{N}\right)-\eta_{l}^{N} M_{l, n}\left(\bar{f}_{l+1, \xi_{l, l}^{j}}^{N}\right)\right\|_{\mathbb{L}_{2 p q^{\prime}}}
\end{aligned}
$$

for any $q, q^{\prime} \geq 1$, with $\frac{1}{q}+\frac{1}{q^{\prime}}=1$.

We observe that, as $\left(\xi_{l, l}^{j},\left(\xi_{l, l}^{i}\right)_{0 \leq i \leq N},\left(\xi_{l, n}^{i}\right)_{0 \leq i \leq N}\right)$ have the same distribution, for any $1 \leq j \leq N$, then for any function $h$ and any $1 \leq j, j^{\prime} \leq N$ we have:

$$
\mathbb{E}\left(h\left(\xi_{l, l}^{j},\left(\xi_{l, l}^{i}\right)_{0 \leq i \leq N},\left(\xi_{l, n}^{i}\right)_{0 \leq i \leq N}\right)\right)=\mathbb{E}\left(h\left(\xi_{l, l}^{j^{\prime}},\left(\xi_{l, l}^{i}\right)_{0 \leq i \leq N},\left(\xi_{l, n}^{i}\right)_{0 \leq i \leq N}\right)\right)
$$

which implies that

$$
\begin{gathered}
\sup _{1 \leq j \leq N}\left\|\eta_{n}^{N}\left(\bar{f}_{l+1, \xi_{l, l}^{j}}^{N}\right)-\eta_{l}^{N} M_{l, n}\left(\bar{f}_{l+1, \xi_{l, l}^{j}}^{N}\right)\right\|_{\mathbb{L}_{2 p q^{\prime}}} \\
=\left\|\eta_{n}^{N}\left(\bar{f}_{l+1, \xi_{l, l}^{j}}^{N}\right)-\eta_{l}^{N} M_{l, n}\left(\bar{f}_{l+1, \xi_{l, l}^{j}}^{N}\right)\right\|_{\mathbb{L}_{2 p q^{\prime}}} .
\end{gathered}
$$

As this equation works for any $1 \leq j \leq N$, in further development we take $j=1$ to simplify the notation.

Using Lemma 5.1, and recalling that $\eta_{l}^{N} M_{l, n}\left(g_{l, x}\right)=\eta_{l}^{N}\left(g_{l, x}\right)$, for any $1 \leq$ $j \leq N$ we prove the almost sure estimate

$$
\begin{aligned}
& \sqrt{N} \mathbb{E}\left(\left|\left[\eta_{n}^{N}-\eta_{l}^{N} M_{l, n}\right]\left(\bar{f}_{l+1, \xi_{l, l}^{1}}^{N}\right)\right|^{2 p q^{\prime}} \mid \mathcal{F}_{l}^{N}\right)^{\frac{1}{2 p q^{\prime}}} \\
& \leq 2 a\left(2 p q^{\prime}\right)(n-l)\left[\eta_{l}^{N} M_{l, n}\left(\left|\bar{f}_{l+1, \xi_{l, l}^{1}}^{N}\right|^{2 p q^{\prime}}\right)\right]^{\frac{1}{2 p q^{\prime}}} \\
& \leq 4 a\left(2 p q^{\prime}\right)(n-l)\left\|f_{l+1}\right\|\left(\eta_{l}^{N} M_{l, n}\left(g_{l, \xi_{l, l}^{1}}\right)\right)^{\frac{1}{2 p q^{\prime}}-1} .
\end{aligned}
$$


This yields that

$$
\begin{aligned}
\sqrt{N} \mathbb{E}\left(\left|\left[\eta_{n}^{N}-\eta_{l}^{N} M_{l, n}\right]\left(\bar{f}_{l+1, \xi_{l, l}^{1}}^{N}\right)\right|^{2 p q^{\prime}} \mid \mathcal{F}_{l}^{N}\right)^{\frac{1}{2 p q^{\prime}}} \\
\leq 4 a\left(2 p q^{\prime}\right)(n-l)\left\|f_{l+1}\right\| \eta_{l}^{N}\left(g_{l, \xi_{l, l}^{1}}\right)^{-1}
\end{aligned}
$$

and therefore

$$
\begin{aligned}
& \sqrt{N}\left\|\eta_{n}^{N}\left(\bar{f}_{l+1, \xi_{l, n}^{i}}^{i}\right)-\eta_{l}^{N} M_{l, n}\left(\bar{f}_{l+1, \xi_{l, n}^{i}}^{N}\right)\right\|_{\mathbb{L}_{2 p q^{\prime}}} \\
& \leq 4 a\left(2 p q^{\prime}\right)(n-l)\left\|f_{l+1}\right\|\left\|\eta_{l}^{N}\left(g_{l, \xi_{l, n}^{i}}\right)^{-1}\right\|_{\mathbb{L}_{q}}^{1 /(2 p)}\left\|\eta_{l}^{N}\left(g_{l, \xi_{l, l}^{1}}\right)^{-1}\right\|_{\mathbb{L}_{2 p q^{\prime}}}
\end{aligned}
$$

Finally, by (6.4), we conclude that

$$
\begin{aligned}
\sqrt{N}\left\|\widehat{M}_{l+1}^{\prime}(f)\left(\xi_{l, n}^{i}\right)-M_{l+1}^{\prime}(f)\left(\xi_{l, n}^{i}\right)\right\|_{\mathbb{L}_{p}} & \\
\leq 4 a\left(2 p q^{\prime}\right)(n-l)\left\|f_{l+1}\right\|\left\|\eta_{n}^{N}\left(g_{l, \xi_{l, n}^{i}}\right)^{-1}\right\|_{\mathbb{L}_{2 p}} & \left\|\eta_{l}^{N}\left(g_{l, \xi_{l, n}^{i}}\right)^{-1}\right\|_{\mathbb{L}_{q}}^{1 /(2 p)} \\
& \times\left\|\eta_{l}^{N}\left(g_{l, \xi_{l, l}^{1}}\right)^{-1}\right\|_{\mathbb{L}_{2 p q^{\prime}}} .
\end{aligned}
$$

We prove (5.13), by taking $q=1+2 p$ and $q^{\prime}=1+1 /(2 p)$ so that $q=2 p q^{\prime} \geq 2 p$

$$
\begin{aligned}
& \sqrt{N}\left\|\widehat{M}_{l+1}^{\prime}(f)\left(\xi_{l, n}^{i}\right)-M_{l+1}^{\prime}(f)\left(\xi_{l, n}^{i}\right)\right\|_{\mathbb{L}_{p}} \\
& \leq 4 a(1+2 p)(n-l)\left\|f_{l+1}\right\| \sup _{l \leq k \leq n}\left\|\eta_{k}^{N}\left(g_{l, \xi_{l, k}^{1}}\right)^{-1}\right\|_{\mathbb{L}_{1+2 p}}^{2+1 /(2 p)} .
\end{aligned}
$$

This end of proof is now a direct consequence of Lemma 5.2

ACKNOWLEDGEMENTS: The third named author was supported by FiME, Laboratoire de Finance des Marchés de l'Energie (Dauphine, CREST, EDF R\&D) www.fime-lab.org.

All the authors are grateful to Laurent Plagne for his useful help in accelerating our numerical simulations implementation. 


\section{References}

[1] A. N. Avramidis and H. Matzinger, Convergence of the Stochastic Mesh Estimator for Pricing Bermudan Options, Journal of Comput. Finance, vol. 7, 73-91 (2004).

[2] V. Bally, G. Pagès and J. Printems, A quantization tree method for pricing and hedging multidimensional American options, Math. Finance, 15(1), pp. 119-168 (2005).

[3] V. Bally and D. Talay, The law of the Euler scheme for stochastic differential equations: I. Convergence rate of the distribution function, Probab. Th. Related Fields, pp. 104, 43-60, (1996).

[4] V. Bally and D. Talay, The law of the Euler scheme for stochastic differential equations: II. Approximation of the density, Monte Carlo Methods Appl., 2, pp. 93-128, (1996).

[5] M. Broadie and P. Glasserman, A Stochastic Mesh Method for Pricing High-Dimensional American Options, Journal of Comput. Finance, vol. 7, pp. 35-72, 2004.

[6] B. Bouchard and N. Touzi, Discrete time approximation and Monte Carlo simulation of backward stochastic differential equations, Stochastic Process Appl. 111, pp. 175-206, 2004.

[7] B. Bouchard and X. Warin, Monte-Carlo valorisation of American options: facts and new algorithms to improve existing methods, To appear in $\mathrm{Nu}$ merical Methods in Finance, ed. R. Carmona, P. Del Moral, P. Hu and N. Oudjane (2011).

[8] J. Bucklew and G. Wise, Multidimensional Asymptotic Quantization Theory with rth Power distortion Measures, IEEE Trans. on Information Theory, Special issue on Quantization, 28, no. 2, pp. 239-247 (1982).

[9] J. Carrière, Valuation of the Early-Exercise Price for Options using Simulations and Nonparametric Regression, Insurance: Math. and Eco., 19 (1), pp. 19-30 (1996).

[10] P. Del Moral, Measure Valued Processes and Interacting Particle Systems. Application to Nonlinear Filtering Problems, Ann. Appl. Probab., vol. 8, no. 2, pp. 1254-1278 (1998).

[11] P. Del Moral, Feynman-Kac formula. Genealogical and interacting particle systems with applications, Probability and its Applications, Springer Verlag, New York (2004).

[12] P. Del Moral and A. Guionnet, On the stability of interacting processes with applications to filtering and genetic algorithms, Annales de l'Institut Henri Poincaré, Vol. 37, No. 2, 155-194 (2001).

[13] P. Del Moral, B. Rémillard and S. Rubenthaler, Monte Carlo approximations of American options, Technical report (00001585-LG), Lab. J.A. Dieudonné, Univ. Nice Sophia-Antipolis (2006). 
[14] P. Del Moral, L. Miclo, F. Patras and S. Rubenthaler, The Convergence to Equilibrium of Neutral Genetic Models, Stochastic Analysis Appl., Volume 28, Issue 1, pp. 123 - 143 (2010).

[15] P. Dupuis and H. Wang, On the convergence from discrete to continuous time in an optimal stopping problem, Ann. Appl. Probab., 15, pp. 1339-1366 (2005).

[16] D. Egloff, Monte Carlo algorithms for optimal stopping and statistical learning, Ann. Appl. Probab., 15, pp. 1 - 37 (2005).

[17] D. Egloff, M. Kohler, and N. Todorovic, A dynamic look-ahead Monte Carlo algorithm for pricing Bermudan options, Ann. Appl. Probab., 17, pp. 1138-1171 (2007).

[18] P. Glasserman, and B. Yu, Number of paths versus number of basis functions in American option pricing, Ann. Appl. Probab., 14, pp. 1-30 (2004).

[19] E. Gobet, J.-P. Lemor, and X. Warin, A regression-based Monte-Carlo method for backward stochastic differential equations, Ann. Appl. Probab., 15, pp. 2172-2202 (2005).

[20] S. Graf and H. Luschgy, Foundations of quantization for probability distributions, Lecture Notes in Math., No. 1730, Springer (2000).

[21] Fr. Le Gland and N. Oudjane, Stability and uniform approximation of nonlinear filters using the Hilbert metric and application to particle filters, Ann. Appl. Probab., Vol. 14, no. 1, 144-187 (2004).

[22] G. Liu and L. J. Hong, Revisit of stochastic mesh method for pricing American options, Operations Research Letters., 37(6), pp. 411-414 (2009).

[23] F. Longstaff and E.S. Schwartz, Valuing American Options by Simulation: A Simple Least Squares Approach, The Review of Financial Studies, 14, pp. 113-147 (2001).

[24] G. Pagès, A space vector quantization method for numerical integration, Journal of Comput. Appl. Math., 89, pp. 1-38 (1997).

[25] G. Pagès and J. Printems, Functional quantization for numerics with an application to option pricing, Monte Carlo Methods Appl., 11(4), pp. 407446 (2005).

[26] G. Pagès, H. Pham and J. Printems. Optimal quantization and applications to numerical problems in finance, Handbook of Computational and Numerical Methods in Finance, ed. S.T. Rachev, Birkhauser, Boston, (2004).

[27] J.N. Tsitsiklis and B. Van Roy, Regression Methods for Pricing Complex American-Style Options, IEEE Transactions on Neural Networks, 12 (4) pp. 694-703.

[28] R. Van Handel, Uniform time average consistency of Monte Carlo particle filters, Stoch. Proc. Appl., 119, 3835-3861 (2009). 
Centre de recherche INRIA Bordeaux - Sud Ouest Domaine Universitaire - 351, cours de la Libération - 33405 Talence Cedex (France)

Centre de recherche INRIA Grenoble - Rhône-Alpes : 655, avenue de l'Europe - 38334 Montbonnot Saint-Ismier Centre de recherche INRIA Lille - Nord Europe : Parc Scientifique de la Haute Borne - 40, avenue Halley - 59650 Villeneuve d'Ascq Centre de recherche INRIA Nancy - Grand Est : LORIA, Technopôle de Nancy-Brabois - Campus scientifique 615, rue du Jardin Botanique - BP 101 - 54602 Villers-lès-Nancy Cedex

Centre de recherche INRIA Paris - Rocquencourt : Domaine de Voluceau - Rocquencourt - BP 105 - 78153 Le Chesnay Cedex

Centre de recherche INRIA Rennes - Bretagne Atlantique : IRISA, Campus universitaire de Beaulieu - 35042 Rennes Cedex Centre de recherche INRIA Saclay - Île-de-France : Parc Orsay Université - ZAC des Vignes : 4, rue Jacques Monod - 91893 Orsay Cedex Centre de recherche INRIA Sophia Antipolis - Méditerranée : 2004, route des Lucioles - BP 93 - 06902 Sophia Antipolis Cedex 